\title{
Wireless Scheduling Design for Optimizing Both Service Regularity and Mean Delay in Heavy-Traffic Regimes
}

\author{
Bin Li, Ruogu Li, and Atilla Eryilmaz
}

\begin{abstract}
We consider the design of throughput-optimal scheduling policies in multi-hop wireless networks that also possess good mean delay performance and provide regular service for all links - critical metrics for real-time applications. To that end, we study a parametric class of maximum-weight type scheduling policies, called Regular Service Guarantee (RSG) Algorithm, where each link weight consists of its own queuelength and a counter that tracks the time since the last service, namely Time-Since-Last-Service (TSLS). The RSG Algorithm not only is throughput-optimal, but also achieves a tradeoff between the service regularity performance and the mean delay, i.e., the service regularity performance of the RSG Algorithm improves at the cost of increasing mean delay.

This motivates us to investigate whether satisfactory service regularity and low mean-delay can be simultaneously achieved by the RSG Algorithm by carefully selecting its design parameter. To that end, we perform a novel Lyapunov-drift based analysis of the steady-state behavior of the stochastic network. Our analysis reveals that the RSG Algorithm can minimize the total mean queue-length to establish mean delay optimality under heavilyloaded conditions as long as the design parameter weighting for the TSLS scales no faster than the order of $\frac{1}{\sqrt[5]{\epsilon}}$, where $\epsilon$ measures the closeness of the network load to the boundary of the capacity region. To the best of our knowledge, this is the first work that provides regular service to all links while also achieving heavytraffic optimality in mean queue-lengths.
\end{abstract}

\section{INTRODUCTION}

Real-time applications, such as voice over IP or live multi-media streaming, are becoming increasingly popular as smart phones proliferate in wireless networks. To support real-time applications, network algorithm design should not only efficiently manage the interference among simultaneous transmissions, but also meet the requirements of Qualityof-Service (QoS) including delay, packet delivery ratio, and jitter. Such QoS requirements, in turn, depend on the higherorder statistics of the arrival and service process, which poses significant challenges for efficient network algorithm design.

In recent years, there has been an increasing understanding on the algorithm design that targets various aspects of QoS, especially packet delivery ratio requirement (e.g., [8], [9], [11])

This work is supported by the NSF grants: CAREER-CNS-0953515, CNSWiFiUS-1456806, and CCSS-EARS-1444026; the QNRF grants: NPRP 091168-2-455 and NPRP 7-923-2-344; and the DTRA grant: HDTRA1-11-16BRCWMD.

An earlier version of this paper has appeared in ACM International Symposium on Mobile Ad Hoc Networking and Computing (MOBIHOC), Bangalore, India, July, 2013 [12].

$\mathrm{Bin} \mathrm{Li}$ is with the Coordinated Science Laboratory, University of Illinois at Urbana-Champaign, Urbana, IL 61801 USA (e-mail: lib@illinois.edu).

Ruogu Li is with ASSIA, Inc., Redwood City, CA 94065 USA (e-mail: lirg03@gmail.com).

Atilla Eryilmaz is with the Department of Electrical and Computer Engineering, The Ohio State University, Columbus, OH 43210 USA (email:eryilmaz.2@osu.edu). and low end-to-end delay (e.g., [2], [25], [24], [6]). However, these QoS metrics do not fully characterize the Quality-ofExperience (QoE) of users in real-time applications in wireless networks. For example, in the network where each individual user wants to watch its video that is delivered by the base station, each mobile user would like to receive the data from the base station regularly. Yet, both the time-varying nature of wireless channels and the scheduling policy significantly affect the regularity of the received data of each mobile user. The traditional scheduling policies aiming to maximize the system throughput (e.g., [21], [16], [10]) or provide various fairness guarantees (e.g., [15], [3], [17], [20] ) at the base station side do not take users' experience into account and thus lead to the high irregularity of the received data of mobile users.

Our work is motivated by the recent advances made in [13], [14] that provide a promising approach for managing this critical QoS metric. In particular, [13] provides a throughputoptimal algorithm that prioritizes service of links with the largest link weight in general network topologies, where each link weight is the weighted-sum of its own queue-length and a counter, namely the time-since-last-service (TSLS), that tracks the time since the last service. This algorithm improves service regularity as its design parameter $\gamma$ weighting for the TSLS increases (see Section III for more details). Yet, increasing $\gamma$ also has an averse effect on the mean delay performance, which is also vital for most applications.

With this motivation, this paper focuses on the tradeoff between the service regularity and the mean delay performance that this class of policies achieves. In particular, we are interested in identifying the range of values for $\gamma$ in which the mean delay performance guarantees can be provided, while the regularity characteristics are preserved. To that end, we build on the recently developed approach of using Lyapunov drifts for the steady-state analysis of queueing networks [4]. The main result emanating from this analysis is the scaling law of $\gamma$ as the system gets more and more heavily loaded so that the algorithm is mean delay optimal among all feasible scheduling policies, and provides the best service regularity among this class of policies. Specifically, we show that the heavy-traffic optimality is preserved as long as $\gamma$ scales $^{1}$ as $O\left(\frac{1}{\sqrt[5]{\epsilon}}\right)$, where $\epsilon$ is the heavy-traffic parameter characterizing the closeness of the arrival rate vector to the boundary of the capacity region.

Our analysis relates to the vast literature on heavy-traffic analysis of queueing networks (for example, [22], [5], [1], [23], [19], [18]), and in particular extends the Lyapunov driftbased approach in [4]. A critical step in most of these results is

\footnotetext{
${ }^{1}$ We say $a_{n}=O\left(b_{n}\right)$ if there exists a $c>0$ such that $\left|a_{n}\right| \leq c\left|b_{n}\right|$ for two real-valued sequences $\left\{a_{n}\right\}$ and $\left\{b_{n}\right\}$.
} 
to establish a state-space collapse along a single dimension, and thus relate the multi-dimensional system operation to a resource-pooled single dimensional system. Our construction also follows such line of argument in broad strokes. However, the new dynamics of the considered class of algorithms require new Lyapunov functions and techniques in establishing their heavy-traffic optimality.

Note on Notation: We use bold and script font of a variable to denote a vector and a set. Also, let $|\mathcal{A}|$ denote the cardinality of the set $\mathcal{A}$. We use $\operatorname{Int}(\mathcal{A})$ to denote the set of interior points of the set $\mathcal{A}$. We use $\langle\mathbf{x}, \mathbf{y}\rangle$ and $\mathbf{x} \cdot \mathbf{y}$ to denote the inner product and component-wise product of the vector $\mathbf{x}$ and $\mathbf{y}$, respectively. We use $\mathrm{x}^{2}$ and $\sqrt{\mathrm{x}}$ to denote the component-wise square and square root of the vector $\mathbf{x}$, respectively. We also use $\preceq, \succeq, \prec, \succ$ to denote component-wise comparison of two vectors, respectively. Let $\|\mathbf{x}\|_{1}$ and $\|\mathbf{x}\|$ denote the $l_{1}$ and $l_{2}$ norm of the vector $\mathbf{x}$, respectively.

\section{SYSTEM MODEL}

We consider a wireless network represented by a graph $\mathcal{G}=$ $(\mathcal{N}, \mathcal{L})$, where $\mathcal{N}$ is the set of nodes and $\mathcal{L}$ is the set of links. $\mathrm{A}$ node represents a wireless transmitter or receiver, while a link represents a pair of transmitter and receiver that are within the transmission range of each other. We use $L \triangleq|\mathcal{L}|$ for convenience. We consider the link-based conflict model, where links conflicting with each other cannot be active at the same time. We call a set of links that can be active simultaneously as a feasible schedule and denote it as $\mathbf{S}[t]=\left(S_{l}[t]\right)_{l \in \mathcal{L}}$, where $S_{l}[t]=1$ if the link $l$ is scheduled in time slot $t$ and $S_{l}[t]=0$, otherwise. Let $\mathcal{S}$ be the set of all feasible schedules.

We capture the channel fading over link $l$ in time slot $t$ via a non-negative-integer-valued random variable $C_{l}[t]$, with strictly positive mean and $C_{l}[t] \leq C_{\max }, \forall l, t$, for some $C_{\max }<\infty$, which measures the maximum amount of service available in slot $t$, if scheduled. We assume that $\{\mathbf{C}[t]=$ $\left.\left(C_{l}[t]\right)_{l=1}^{L}\right\}_{t \geq 0}$ is an independently and identically distributed (i.i.d.) sequence of random vectors with $\psi_{\mathbf{c}} \triangleq \operatorname{Pr}\{\mathbf{C}[t]=\mathbf{c}\}$. We use $\mathcal{C}$ to denote the set of all possible channel states. Note that $|\mathcal{C}|$ is finite. Let $\mathcal{S}^{(\mathbf{c})} \triangleq\{\mathbf{S} \cdot \mathbf{c}: \mathbf{S} \in \mathcal{S}\}$ denote the set of feasible rate vectors in the channel state $\mathbf{c} \in \mathcal{C}$. Then, the capacity region is defined as $\mathcal{R} \triangleq \sum_{\mathbf{c}} \psi_{\mathbf{c}} \cdot \operatorname{Conv}\left\{\mathcal{S}^{(\mathbf{c})}\right\}$, where $\operatorname{Conv}\{\mathcal{A}\}$ denotes the convex hull of the set $\mathcal{A}$.

We assume a per-link traffic model, where $A_{l}[t]$ denotes the number of packets arriving at link $l$ in slot $t$ that are independently distributed over links and i.i.d. over time with finite mean $\lambda_{l}>0$, and $A_{l}[t] \leq A_{\max }, \forall l, t$, for some $A_{\max }<\infty$. Accordingly, a queue is maintained for each link $l$ with $Q_{l}[t]$ denoting its queue length at the beginning of time slot $t$. Let $U_{l}[t]=\max \left\{0, C_{l}[t] S_{l}[t]-Q_{l}[t]-A_{l}[t]\right\}$ be the unused service for queue $l$ in slot $t$. Then, the evolution of queue $l$ is described as follows:

$$
Q_{l}[t+1]=Q_{l}[t]+A_{l}[t]-C_{l}[t] S_{l}[t]+U_{l}[t], \quad \forall l .
$$

We say that the queue $l$ is strongly stable if it satisfies

$$
\limsup _{T \rightarrow \infty} \frac{1}{T} \sum_{t=1}^{T} \mathbb{E}\left[Q_{l}[t]\right]<\infty .
$$

We call an algorithm throughput-optimal if it makes all queues strongly stable for any arrival rate vector $\boldsymbol{\lambda}=\left(\lambda_{l}\right)_{l=1}^{L}$ that lies strictly within the capacity region.

Our goal is to design a throughput-optimal scheduling algorithm that also possesses the following desirable properties for satisfying the QoS requirements: (i) provides regular services in the sense that the normalized second-moment of the interservice times of the links is small; and (ii) achieves low mean delay in the sense that the total mean queue-lengths is small, especially in the regime where the system is heavily-loaded when delay effects are most pronounced.

Next, we provide a regular service scheduler that possesses throughput-optimality and regular service guarantees, and then investigate its mean-delay performance under the heavy-traffic regime.

\section{Regular Service Scheduler}

One of our goals is to provide regular services for each link, which is related to the second moment of the inter-service times. To characterize the inter-service time, we introduce a counter $T_{l}$ for each link $l$, namely Time-Since-Last-Service (TSLS), to keep track of the time since link $l$ was last served. In particular, each $T_{l}$ increases by 1 in each time slot when link $l$ has zero transmission rate, either because it is not scheduled, or because its channel is unavailable, i.e., $C_{l}[t]=0$, and drops to 0 , otherwise. More precisely, the evolution of $T_{l}$ is described as follows:

$$
T_{l}[t+1]= \begin{cases}0 & \text { if } S_{l}[t] C_{l}[t]>0 \\ T_{l}[t]+1 & \text { if } S_{l}[t] C_{l}[t]=0\end{cases}
$$

Thus, the TSLS records the link "age" since the last time it received service, and is closely related to the inter-service time. Indeed, in [13], we showed that the normalized second moment of the inter-service times of each link is proportional to the mean value of its TSLS for any stabilizing policy. Thus, the TSLS has a direct impact on service regularity: the smaller the mean TSLS value, the more regular the service.

This connection motivates the following maximum-weight type algorithm that uses a combination of queue-lengths and TSLS values as its weights:

\section{Regular Service Guarantee (RSG) Algorithm²}

In each time slot $t$, select a schedule $\mathbf{S}^{*}[t]$ such that

$$
\mathbf{S}^{*}[t] \in \underset{\mathbf{S} \in \mathcal{S}}{\arg \max } \sum_{l=1}^{L}\left(\alpha_{l} Q_{l}[t]+\gamma \beta_{l} T_{l}[t]\right) C_{l}[t] S_{l},
$$

where $\alpha_{l}>0, \beta_{l} \geq 0$ and $\gamma \geq 0$ are design parameters.

The parameters $\left(\alpha_{l}\right)_{l=1}^{L}$ are weighting factors for the queuelengths, where a larger $\alpha_{l}$ will result in a smaller average queue-length. The parameters $\left(\beta_{l}\right)_{l=1}^{L}$ weigh $T_{l}[t]$ differently

\footnotetext{
${ }^{2}$ For wireless networks with multi-hop traffic, where packets may traverse multiple links before their departure, we maintain queue-length and TSLS counter for each flow at each link. Then, we propose a backpressure-type algorithm with the link weight consisting of queue-length and TSLS. We can still show that the proposed backpressure-type algorithm achieves maximum throughput. However, the service regularity of each flow is hard to characterize in this setup, which is left for future research.
} 
for each link $l$, with $\gamma$ being a common scaling factor for all links. Note that the RSG Algorithm coincides with the MWS Algorithm when $\gamma=0$. Yet, the true significance of the RSG algorithm is observed for large $\gamma$, since as $\gamma$ increases, the RSG Algorithm prioritizes the schedule with the larger TSLS, hence providing more regular services for each link. In [13], we have showed that the RSG Algorithm not only achieves throughput optimality but also provides regular service guarantees.

Yet, large values of $\gamma$ may also deteriorate the mean delay performance. We demonstrate this tradeoff in a single-hop non-fading network with 4 links, where the number of packets arriving at each link follows a Bernoulli distribution with the arrival rate of 0.225 . Let $\alpha_{l}=\beta_{l}=1$ for each link $l$. Figure 1 shows the mean delay and service regularity performance of the RSG Algorithm with varying $\gamma$.

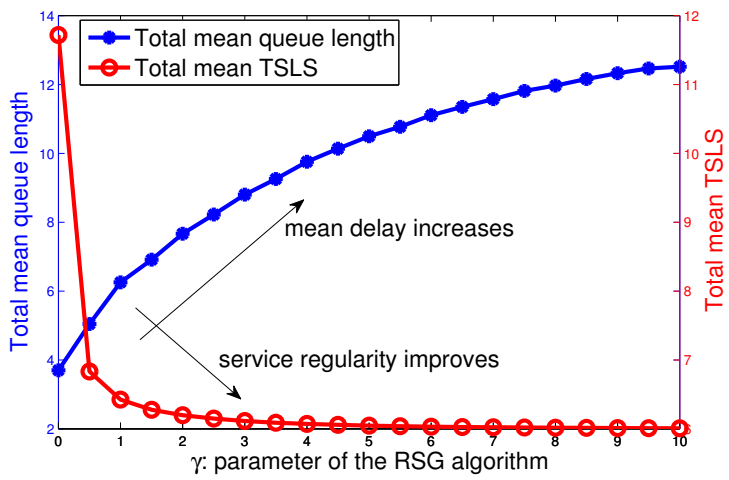

Fig. 1: Performance of the RSG Algorithm

Figure 1 reveals that the improved service regularity of the RSG Algorithm with increasing $\gamma$ comes at the cost of larger mean delays. We can show that the mean of the total TSLS value is minimized as $\gamma$ goes to $\infty$ (see [13]). On the other hand, it is known (e.g. [19], [4]) that the mean queue-lengths are minimized under heavily-loaded conditions (cf. Section IV for more detail) when $\gamma=0$. In view of the tradeoff observed in the above figure, our objective is to understand whether both the regularity and the mean-delay optimality characteristics of the RSG Algorithm can be preserved, especially under heavilyloaded conditions, by carefully selecting $\gamma$.

In the next section, we answer this question in the affirmative by explicitly characterizing how $\gamma$ should scale with respect to the traffic load in order to achieve the heavytraffic optimality while also optimizing the service regularity performance of the RSG Algorithm.

\section{HeAvy-TRAFFiC Optimality Result}

In this section, we present our main result for the RSG Algorithm in terms of its mean delay optimality under the heavy-traffic limit, where the arrival rate vector approaches the boundary of the capacity region.
In the rest of paper, we consider the RSG Algorithm ${ }^{3}$ with $\alpha_{l}=1, \forall l$. We first note that the capacity region $\mathcal{R}$ is a polyhedron due to the discreteness and finiteness of the service rate choices, and thus has a finite number of faces. We consider the exogenous arrival process $\left\{\mathbf{A}^{(\epsilon)}[t]\right\}_{t \geq 0}$ with mean rate vector $\boldsymbol{\lambda}^{(\epsilon)} \in \operatorname{Int}(\mathcal{R})$, where $\epsilon$ measures the Euclidean distance of $\boldsymbol{\lambda}^{(\epsilon)}$ to the boundary of $\mathcal{R}$ (see Figure 2).

In heavy-traffic analysis, we study the system performance as $\epsilon$ decreases to zero, i.e., as the arrival rate vector approaches $\lambda^{(0)}$ belonging to the relative interior of a face, referred to as the dominant hyperplane $\mathcal{H}^{(\mathbf{d})}$. We denote $\mathcal{H}^{(\mathbf{d})} \triangleq\left\{\mathbf{r} \in \mathbb{R}^{L}\right.$ : $\langle\mathbf{r}, \mathbf{d}\rangle=b\}$, where $b \in \mathbb{R}$, and $\mathbf{d} \in \mathbb{R}^{L}$ is the normal vector of the hyperplane $\mathcal{H}^{(\mathbf{d})}$ satisfying $\|\mathbf{d}\|=1$ and $\mathbf{d} \succeq \mathbf{0}$.

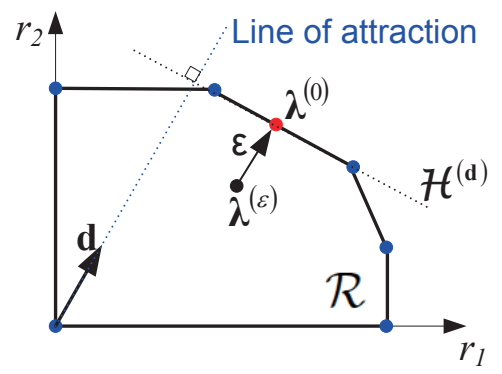

Fig. 2: Geometric structure of capacity region

We are interested in understanding the steady-state queuelength values with vanishing $\epsilon$. To that end, we first provide a generic lower bound for all feasible schedulers by constructing a hypothetical single-server queue with the arrival process $\left\langle\mathbf{d}, \mathbf{A}^{(\epsilon)}[t]\right\rangle$, and the i.i.d service process $\Psi[t]$ with the probability distribution

$$
\operatorname{Pr}\left\{\Psi[t]=b_{\mathbf{c}}\right\}=\psi_{\mathbf{c}}, \quad \text { for each channel state } \mathbf{c} \in \mathcal{C},
$$

where $b_{\mathbf{c}} \triangleq \max _{\mathbf{s} \in \mathcal{S}(\mathbf{c})}\langle\mathbf{d}, \mathbf{c} \cdot \mathbf{s}\rangle$ is the maximum $\mathbf{d}$-weighted service rate achievable in channel state $\mathbf{c} \in \mathcal{C}$. By the construction of capacity region $\mathcal{R}$, we have $\mathbb{E}[\Psi[t]]=b$. Also, it is easy to show that the constructed single-server queuelength $\{\Phi[t]\}_{t \geq 0}$ is stochastically smaller than the queuelength process $\left\{\left\langle\mathbf{d}, \mathbf{Q}^{(\epsilon)}[t]\right\rangle\right\}_{t \geq 0}$ under any feasible scheduling policy. Hence, by using [4, Lemma 4], we have the following

${ }^{3}$ Let $A_{l}^{\prime}[t] \triangleq \sqrt{\alpha_{l}} A_{l}[t]$ and $C_{l}^{\prime}[t] \triangleq \sqrt{\alpha_{l}} C_{l}[t], \forall t \geq 0, \forall l$. Also let $\mathcal{S}^{\prime(\mathbf{c})} \triangleq\{\sqrt{\boldsymbol{\alpha}} \cdot \mathbf{c} \cdot \mathbf{S}: \mathbf{S} \in \mathcal{S}\}$ and $\mathcal{R}^{\prime} \triangleq \sum_{\mathbf{c}} \psi_{\mathbf{c}} \cdot \mathrm{CH}\left\{\mathcal{S}^{\prime(\mathbf{c})}\right\}$. Construct a hypothetical system with the arrival process $\left\{\mathbf{A}^{\prime}[t]=\left(A_{l}^{\prime}[t]\right)_{l=1}^{L}\right\}_{t \geq 0}$ and the channel fading process $\left\{\mathbf{C}^{\prime}[t]=\left(C_{l}^{\prime}[t]\right)_{l=1}^{L}\right\}_{t \geq 0}$ under the following RSG Algorithm in the hypothetical system:

$$
\mathbf{S}^{* *}[t] \in \underset{\mathbf{S} \in \mathcal{S}}{\arg \max } \sum_{l=1}^{L}\left(Q_{l}^{\prime}[t]+\gamma \frac{\beta_{l}}{\sqrt{\alpha_{l}}} T_{l}^{\prime}[t]\right) C_{l}^{\prime}[t] S_{l},
$$

where $T_{l}^{\prime}$ is the TSLS counter for link $l$ in the hypothetical system and evolves as follows:

$$
T_{l}^{\prime}[t+1]= \begin{cases}0 & \text { if } S_{l}^{\prime}[t] C_{l}^{\prime}[t]>0 \\ T_{l}^{\prime}[t]+1 & \text { if } S_{l}^{\prime}[t] C_{l}^{\prime}[t]=0\end{cases}
$$

Let $\left\{\mathbf{Q}[t]=\left(Q_{l}[t]\right)_{l=1}^{L}\right\}_{t \geq 0}$ and $\left\{\mathbf{Q}^{\prime}[t]=\left(Q_{l}^{\prime}[t]\right)_{l=1}^{L}\right\}_{t \geq 0}$ be the queuelength process under the RSG Algorithm in the original system and the RSG Algorithm with $\alpha_{l}=1$ for each link $l$ in the hypothetical system, respectively. Then, it is easy to show that $\{\sqrt{\boldsymbol{\alpha}} \cdot \mathbf{Q}[t]\}_{t \geq 0}$ is stochastically equal to $\left\{\mathbf{Q}^{\prime}[t]\right\}_{t \geq 0}$. Thus, we can study the queue length behavior under the RSG Algorithm with $\alpha_{l}=1$ for each link $l$ in the hypothetical system. 
lower bound on the expected steady-state queue-length under any feasible scheduling policy.

Proposition 1: Let $\overline{\mathbf{Q}}^{(\epsilon)}$ be a random vector with the same distribution as the steady-state distribution of the queue length processes under any feasible scheduling policy. Consider the heavy-traffic limit $\epsilon \downarrow 0$, suppose that the variance vector $\left(\boldsymbol{\sigma}^{(\epsilon)}\right)^{2}$ of the arrival process $\left\{\mathbf{A}^{(\epsilon)}[t]\right\}_{t \geq 0}$ converges to a constant vector $\sigma^{2}$. Then,

$$
\lim _{\epsilon \downarrow 0} \epsilon \mathbb{E}\left[\left\langle\mathbf{d}, \overline{\mathbf{Q}}^{(\epsilon)}\right\rangle\right] \geq \frac{\zeta}{2},
$$

where $\zeta \triangleq\left\langle\mathbf{d}^{2}, \boldsymbol{\sigma}^{2}\right\rangle+\operatorname{Var}(\Psi)$.

This fundamental lower bound of all feasible scheduling policies motivates the following definition of heavy-traffic optimality of a scheduler.

Definition 1: (Heavy-Traffic Optimality) A scheduler is called heavy-traffic optimal, if its steady-state queue length vector $\overline{\mathbf{Q}}^{(\epsilon)}$ satisfies

$$
\lim _{\epsilon \downarrow 0} \epsilon \mathbb{E}\left[\left\langle\mathbf{d}, \overline{\mathbf{Q}}^{(\epsilon)}\right\rangle\right] \leq \frac{\zeta}{2},
$$

where $\zeta$ is defined in Proposition 1.

It is well-known that the MWS Algorithm, which corresponds to the RSG Algorithm with $\gamma=0$, is heavy-traffic optimal (e.g., [19], [4]). This is shown by first establishing a state-space collapse, i.e., the deviations of queue lengths from the direction $\mathbf{d}$ are bounded, independent of heavy-traffic parameter $\epsilon$. Since the lower bound of mean queue length is of order of $\frac{1}{\epsilon}$, the deviations from the direction $\mathbf{d}$ are negligible compared to the large queue length for a sufficiently small $\epsilon$, and thus the queue lengths concentrate along the normal vector $\mathbf{d}$. Because of this, we also call the normal vector $\mathbf{d}$ the line of attraction.

However, as discussed in Section III, we are interested in large values of $\gamma$ to provide satisfactory service regularity. Yet, it is unknown whether the RSG Algorithm can remain heavy-traffic optimal when $\gamma$ is non-zero, since larger values of $\gamma$ leads to higher mean queue-lengths (cf. Figure 1). Also, the state-space collapse result is not applicable since the deviations from the line of attraction depend on $\gamma$. This raises the question of how $\gamma(\epsilon)$ should scale with $\epsilon$ in order to achieve heavy-traffic optimality while allowing $\gamma(\epsilon)$ to take large values (providing more regular services). We answer this interesting and challenging question by providing the following main result, proved in Section VI.

Proposition 2: Let $\overline{\mathbf{Q}}^{(\epsilon)}$ be a random vector with the same distribution as the steady-state distribution of the queue length processes under the RSG Algorithm. Consider the heavy-traffic limit $\epsilon \downarrow 0$, suppose that the variance vector $\left(\boldsymbol{\sigma}^{(\epsilon)}\right)^{2}$ of the arrival process $\left\{\mathbf{A}^{(\epsilon)}[t]\right\}_{t \geq 0}$ converges to a constant vector $\sigma^{2}$. Suppose the channel fading satisfies the mild assumption ${ }^{4}$ $\operatorname{Pr}\left\{C_{l}[t]=0\right\}>0$, for all $l \in \mathcal{L}$. Then,

$$
\epsilon \mathbb{E}\left[\left\langle\mathbf{d}, \overline{\mathbf{Q}}^{(\epsilon)}\right\rangle\right] \leq \frac{\zeta^{(\epsilon)}}{2}+\bar{B}^{(\epsilon)},
$$

\footnotetext{
${ }^{4}$ We note that our result holds in single-hop network topologies without this assumption, and its extension to more general settings is part of our future work.
}

where $\zeta^{(\epsilon)} \triangleq\left\langle\mathbf{d}^{2},\left(\boldsymbol{\sigma}^{(\epsilon)}\right)^{2}\right\rangle+\operatorname{Var}(\Psi)+\epsilon^{2}$ and $\bar{B}^{(\epsilon)}$ is defined in (23).

Further, if $\gamma(\epsilon)=O\left(\frac{1}{\sqrt[5]{\epsilon}}\right)$, then $\lim _{\epsilon \downarrow 0} \bar{B}^{(\epsilon)}=0$ and thus the RSG Algorithm is heavy-traffic optimal.

This result is interesting in that it provides an explicit scaling regime in which the design parameter $\gamma(\epsilon)$ can be increased to utilize the service regulating nature of the RSG Algorithm without sacrificing the heavy-traffic optimality. Intuitively, if $\gamma(\epsilon)$ scales slowly as $\epsilon$ vanishes, each link weight is dominated by its own queue length in the heavytraffic regime and thus the heavy-traffic optimality may be maintained; otherwise, the heavy-traffic optimality result may not hold, as will be demonstrated in the next section.

\section{Simulation Results}

In this section, we provide simulation results to compare the mean delay and service regularity performance of the RSG Algorithm with the MWS Algorithm. In the simulation, we consider a single-hop non-fading network with 4 links. Its capacity region is $\mathcal{R}=\left\{\boldsymbol{\lambda}=\left(\lambda_{l}\right)_{l=1}^{4} \succeq \mathbf{0}: \sum_{l=1}^{4} \lambda_{l}<1\right\}$. We use arrival process where the number of arrivals in each slot follows a Bernoulli distribution. We consider the symmetric case $\boldsymbol{\lambda}^{(\epsilon)}=\left(1-\frac{\epsilon}{2}\right) \times\left[\frac{1}{4}, \frac{1}{4}, \frac{1}{4}, \frac{1}{4}\right]$, and the asymmetric case $\boldsymbol{\lambda}^{(\epsilon)}=\left[\frac{1}{2}, \frac{1}{4}, \frac{1}{8}, \frac{1}{16}\right]+\left(1-\frac{\epsilon}{32}\right) \times\left[\frac{1}{64}, \frac{1}{64}, \frac{1}{64}, \frac{1}{64}\right]$.

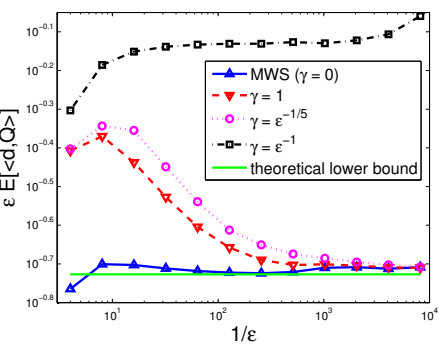

(a) Mean queue length

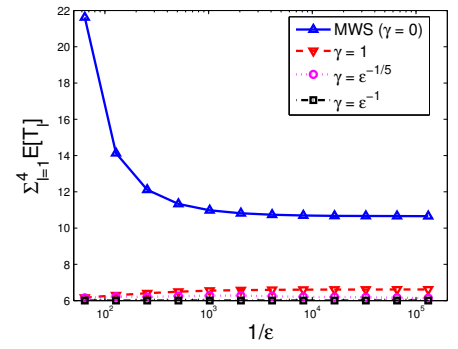

(b) Service regularity
Fig. 3: Symmetric arrivals in a single-hop network

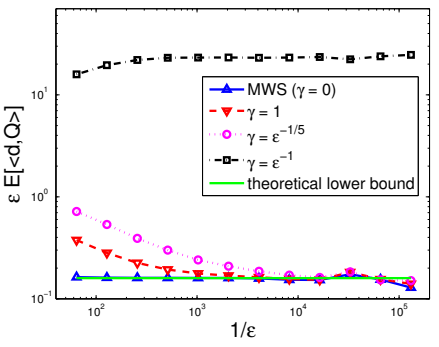

(a) Mean queue length

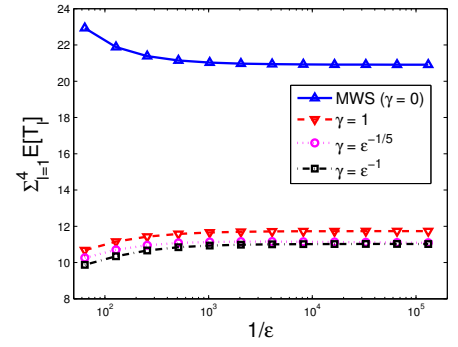

(b) Service regularity
Fig. 4: Asymmetric arrivals in a single-hop network

From Figure $3 \mathrm{a}$ and $4 \mathrm{a}$, we can observe that the RSG Algorithm with both $\gamma=1$ and $\gamma=\frac{1}{\sqrt[5]{\epsilon}}$, and the MWS Algorithm converge to the theoretical lower bound and thus is heavy-traffic optimal, which confirms our theoretical results. Yet, the RSG Algorithm with $\gamma=\frac{1}{\epsilon}$ has large mean queue length, which does not match with the theoretical lower bound 
and thus is not heavy-traffic optimal. Hence, $\gamma$ should scale as slowly as $O\left(\frac{1}{\sqrt[5]{\epsilon}}\right)$ to preserve heavy-traffic optimality.

From Figure $3 \mathrm{~b}$ and $4 \mathrm{~b}$, we can see that the RSG Algorithm with even $\gamma=1$ significantly outperforms the MWS Algorithm in terms of service regularity. More remarkably, the RSG Algorithm with $\gamma=\frac{1}{\sqrt[5]{\epsilon}}$ can achieve the lower bound (see [13]) achieved by the round robin policy under symmetric arrivals.

\section{Vi. Detailed Heavy-Traffic Analysis}

In this section, we prove Proposition 2 by using the analytical approach in [4], which includes two parts: (i) showing state-space collapse; (ii) using the state-space collapse result to obtain an upper bound on the mean queue lengths. Yet, it is worth noting that the abrupt dynamics of TSLS counters poses significant challenges in heavy-traffic analysis. In particular, it requires new Lyapunov functions and a novel technique to establish heavy-traffic optimality of the RSG Algorithm.

\section{A. State-Space Collapse}

In this subsection, we establish a state-space collapse result under the RSG Algorithm. That is, we develop upper bounds for the deviation of steady-state queue-lengths from the line of attraction $\mathbf{d}$ and TSLS counters. These upper bounds are crucial to establish our main result.

We have mentioned in Section III that the RSG Algorithm is throughput-optimal, i.e., it stabilizes all queues for any arrival rate vector that are strictly within the capacity region. Let $\left\{\mathbf{Q}^{(\epsilon)}[t]\right\}_{t \geq 0}$ and $\left\{\mathbf{T}^{(\epsilon)}[t]\right\}_{t \geq 0}$ be queue-length processes and TSLS counters under the RSG Algorithm, respectively. Also, we use $\overline{\mathbf{Q}}^{(\epsilon)}$ and $\overline{\mathbf{T}}^{(\epsilon)}$ to denote their steady-state queue-length random vector and TSLS random vector, respectively. Then, by the continuous mapping theorem, we have

$$
\begin{array}{rlrl}
\mathbf{Q}_{\|}^{(\epsilon)}[t] & \Rightarrow \overline{\mathbf{Q}}_{\|}^{(\epsilon)}, & & \mathbf{Q}_{\perp}^{(\epsilon)}[t] \Rightarrow \overline{\mathbf{Q}}_{\perp}^{(\epsilon)} ; \\
\mathbf{T}_{\|}^{(\epsilon)}[t] \Rightarrow \overline{\mathbf{T}}_{\|}^{(\epsilon)}, & & \mathbf{T}_{\perp}^{(\epsilon)}[t] \Rightarrow \overline{\mathbf{T}}_{\perp}^{(\epsilon)},
\end{array}
$$

where $\Rightarrow$ denotes convergence in distribution, and we define the projection and the perpendicular vector of any given $L$ dimensional vector $\mathbf{I}$ with respect to the normal vector $\mathbf{d}$ as:

$$
\mathbf{I}_{\|} \triangleq\langle\mathbf{d}, \mathbf{I}\rangle \mathbf{d}, \quad \mathbf{I}_{\perp} \triangleq \mathbf{I}-\mathbf{I}_{\|} .
$$

Next, we will show that under the RSG Algorithm, the second moment of $\left\|\overline{\mathbf{Q}}_{\perp}^{(\epsilon)}\right\|$ is bounded, dependent on $\gamma(\epsilon)$, while the second moment of $\left\|\overline{\mathbf{T}}^{(\epsilon)}\right\|$ is bounded by some constant independent of $\epsilon$. Noting that the mean queue-length under any policy scales at least the order of $1 / \epsilon$ by Proposition 1 , the state-space collapse happens in the following sense: by carefully selecting the scaling law of $\gamma(\epsilon)$ with respect to $\epsilon$, both $\mathbb{E}\left[\left\|\overline{\mathbf{Q}}_{\perp}^{(\epsilon)}\right\|^{2}\right]$ and $\mathbb{E}\left[\left\|\overline{\mathbf{T}}^{(\epsilon)}\right\|^{2}\right]$ are negligible compared to the large mean queue-length for a sufficiently small $\epsilon$.

Proposition 3: If $\operatorname{Pr}\left\{C_{l}[t]=0\right\}>0, \forall l \in \mathcal{L}$, then, under the RSG Algorithm, there exists a constant $N_{T, 2}$, independent of $\epsilon$, such that

$$
\mathbb{E}\left[\left\|\overline{\mathbf{Q}}_{\perp}^{(\epsilon)}\right\|^{2}\right]=O\left((\gamma(\epsilon))^{4}(\log \gamma(\epsilon))^{2}\right),
$$

$$
\mathbb{E}\left[\left\|\boldsymbol{\beta} \cdot \overline{\mathbf{T}}^{(\epsilon)}\right\|^{2}\right] \leq N_{T, 2},
$$

where we recall that $\boldsymbol{\beta} \triangleq\left(\beta_{l}\right)_{l \in \mathcal{L}}$.

It is quite challenging to directly give an upper bound on $\mathbb{E}\left[\left\|\overline{\mathbf{Q}}_{\perp}^{(\epsilon)}\right\|^{2}\right]$. Instead, we will first upper-bound the moment generation function of $\left\|\overline{\mathbf{Q}}_{\perp}^{(\epsilon)}\right\|$, and then use the relationship between the moments of a random variable and its moment generation function to upper-bound $\mathbb{E}\left[\left\|\overline{\mathbf{Q}}_{\perp}^{(\epsilon)}\right\|^{2}\right]$.

In order to obtain an upper bound on the moment generation function of $\left\|\overline{\mathbf{Q}}_{\perp}^{(\epsilon)}\right\|$, we first study the drift of the Lyapunov function

$$
V_{\perp}\left(\mathbf{Q}^{(\epsilon)}, \mathbf{T}^{(\epsilon)}\right) \triangleq\left\|\left(\mathbf{Q}_{\perp}^{(\epsilon)}, \sqrt{2 \gamma(\epsilon) C_{\max } \boldsymbol{\beta} \cdot \mathbf{T}^{(\epsilon)}}\right)\right\|,
$$

and show that when $V_{\perp}\left(\mathbf{Q}^{(\epsilon)}, \mathbf{T}^{(\epsilon)}\right)$ is sufficiently large, it has a strictly negative drift independent of $\epsilon$, which is characterized in the following key lemma.

Lemma 1: Under the RSG Algorithm, there exist positive constants $\kappa$ and $\varsigma$, independent of $\epsilon$, such that whenever $V_{\perp}\left(\mathbf{Q}^{(\epsilon)}[t], \mathbf{T}^{(\epsilon)}[t]\right)>\kappa$, we have

$$
\mathbb{E}\left[\Delta V_{\perp}\left(\mathbf{Q}^{(\epsilon)}[t], \mathbf{T}^{(\epsilon)}[t]\right) \mid \mathbf{Q}^{(\epsilon)}[t], \boldsymbol{T}^{(\epsilon)}[t]\right]<-\varsigma,
$$

where $\Delta V_{\perp}\left(\mathbf{Q}^{(\epsilon)}[t], \mathbf{T}^{(\epsilon)}[t]\right) \triangleq V_{\perp}\left(\mathbf{Q}^{(\epsilon)}[t+1], \mathbf{T}^{(\epsilon)}[t+1]\right)-$ $V_{\perp}\left(\mathbf{Q}^{(\epsilon)}[t], \mathbf{T}^{(\epsilon)}[t]\right)$.

The proof of Lemma 1 is available in Appendix A.

In [4], after showing that $\left\|\overline{\mathbf{Q}}_{\perp}^{(\epsilon)}\right\|$ has a negative drift under the MWS Algorithm when $\left\|\overline{\mathbf{Q}}_{\perp}^{(\epsilon)}\right\|$ is large enough and observing that the drift of $\left\|\overline{\mathbf{Q}}_{\perp}^{(\epsilon)}\right\|$ is bounded, the authors utilize [7, Theorem 2.3] to develop an upper bound on the moment generation function of $\left\|\overline{\mathbf{Q}}_{\perp}^{(\epsilon)}\right\|$, which implies the existence of all moments of $\left\|\overline{\mathbf{Q}}_{\perp}^{(\epsilon)}\right\|$. However, the absolute value of the drift $\Delta V_{\perp}\left(\mathbf{Q}^{(\epsilon)}, \mathbf{T}^{(\epsilon)}\right)$ has neither an upper bound nor an exponential tail given the current system state $\left(\mathbf{Q}^{(\epsilon)}, \mathbf{T}^{(\epsilon)}\right)$, since the TSLS counters have bounded increment but unbounded decrement, i.e., they can at almost increase by 1 and drop to 0 once their corresponding links are scheduled. Therefore, we cannot directly apply [7, Theorem 2.3], which requires either boundedness or the exponential tail of the Lyapunov drift to establish the existence of the moment generation function of the system-state variables in addition to Lemma 1. Indeed, for a Markov Chain with a strictly negative drift of Lyapunov function, if its Lyapunov drift has bounded increment but unbounded decrement, its moment generation function may not exist.

Counterexample: Consider a Markov Chain $\{X[t]\}_{t \geq 0}$ with the following transition probability:

$$
P_{j, j+1}=\left\{\begin{array}{ll}
1 & \text { if } j=0 ; \\
\frac{1}{2} & \text { if } j=1 ; \\
\frac{j-1}{j+1} & \text { if } j \geq 2 .
\end{array} \quad P_{j, 0}= \begin{cases}\frac{1}{2} & \text { if } j=1 ; \\
\frac{2}{j+1} & \text { if } j \geq 1 .\end{cases}\right.
$$

The state transition diagram of Markov Chain $\{X[t]\}_{t \geq 0}$ is shown in Figure 5. Consider a linear Lyapunov function $X$. For any $X \geq 2$, we have

$$
\mathbb{E}[X[t+1]-X[t] \mid X[t]=X]=\frac{X-1}{X+1}-\frac{2 X}{X+1}=-1 .
$$

Thus, the Lyapunov function $X$ has a strictly negative drift 


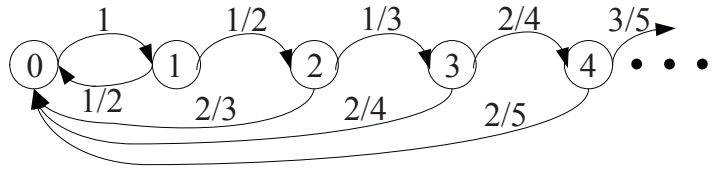

Fig. 5: Markov Chain $\{X[t]\}_{t \geq 0}$

when $X \geq 2$ and hence the steady-state distribution of the Markov Chain exists. Recall that its drift increases at almost by 1 , but has unbounded decrement, which has similar dynamics with the system under the RSG Algorithm.

Next, we will show that even the first moment of this Markov Chain does not exist, let alone its moment generation function. Let $\bar{X}$ be the steady-state random variable of the Markov Chain and $\pi_{j} \triangleq \operatorname{Pr}\{\bar{X}=j\}$. According to the global balance equations, we can easily calculate

$$
\pi_{1}=\pi_{0}=\frac{1}{3}, \quad \pi_{j}=\frac{1}{3 j(j-1)}, \quad \forall j \geq 2 .
$$

Thus, we have

$$
\mathbb{E}[\bar{X}]=\sum_{j=1}^{\infty} j \pi_{j}=\frac{1}{3}+\sum_{j=2}^{\infty} \frac{1}{3(j-1)}=\infty .
$$

Fortunately, we can establish an upper bound on the moment generation function of $\left\|\overline{\mathbf{Q}}_{\perp}^{(\epsilon)}\right\|$ under the RSG Algorithm by exploiting the coupling between queue length processes and TSLS counters under the RSG Algorithm. Here, we need a mild assumption that $\operatorname{Pr}\left\{C_{l}[t]=0\right\}>0, \forall l \in \mathcal{L}$, which leads to the following lemma that all TSLS counters have an exponential tail independent of $\epsilon$.

Lemma 2: If $p_{l} \triangleq \operatorname{Pr}\left\{C_{l}[t]=0\right\}>0, \forall l \in \mathcal{L}$, then, under the RSG Algorithm, there exists a $\vartheta \in(0,1)$, independent of $\epsilon$, such that

$$
\operatorname{Pr}\left\{T_{l}[t] \geq m\right\} \leq \vartheta^{m}, \quad \forall t \geq m \geq 0, \forall l \in \mathcal{L} .
$$

The proof of Lemma 2 is available in Appendix B.

Remark: We can also show that all TSLS counters still have an exponential tail independent of $\epsilon$ in non-fading single-hop network topologies. The extension to the more general setup is left for future research.

Lemma 2 directly implies (11). The rest of proof mainly builds on the analytical technique in [7], while it requires carefully partitioning the space $\left(\mathbf{Q}_{\perp}^{(\epsilon)}, \mathbf{T}^{(\epsilon)}\right)$ and exploiting the coupling between the queue-length processes and TSLS counters. The detailed proof can be found in Appendix C.

\section{B. Proof of Main Result}

Having established the state-space collapse result, we are ready to show the heavy-traffic optimality of the RSG Algorithm. In this subsection, we first give an upper bound on $\mathbb{E}\left[\left\langle\mathbf{d}, \overline{\mathbf{Q}}^{(\epsilon)}\right\rangle\right]$ under the RSG Algorithm and then establish its heavy-traffic optimality by selecting $\gamma(\epsilon)=O\left(\frac{1}{\sqrt[5]{\epsilon}}\right)$. In [13], we have shown that all moments of steady-state system variables, such as queue-lengths and TSLS, are bounded under the RSG Algorithm, which enables us to analyze its heavy- traffic performance by using the methodology of "setting the drift of a Lyapunov function equal to zero".

We will omit the superscript $\epsilon$ associated with the queue lengths and TSLS counters for brevity in the rest of proof. In order to derive an upper bound on $\mathbb{E}\left[\left\langle\mathbf{d}, \overline{\mathbf{Q}}^{(\epsilon)}\right\rangle\right]$, we need the following fundamental identity (see [4, Lemma 8$]$ ):

$$
\begin{aligned}
& \frac{\mathbb{E}\left[\langle\mathbf{d}, \mathbf{U}(\overline{\mathbf{Q}}, \overline{\mathbf{T}}, \mathbf{C})\rangle^{2}\right]}{2}+\frac{\mathbb{E}\left[\left\langle\mathbf{d}, \mathbf{A}-\mathbf{C} \cdot \mathbf{S}^{*}(\overline{\mathbf{Q}}, \overline{\mathbf{T}}, \mathbf{C})\right\rangle^{2}\right]}{2} \\
+ & \mathbb{E}\left[\left\langle\mathbf{d}, \overline{\mathbf{Q}}+\mathbf{A}-\mathbf{C} \cdot \mathbf{S}^{*}(\overline{\mathbf{Q}}, \overline{\mathbf{T}}, \mathbf{C})\right\rangle\langle\mathbf{d}, \mathbf{U}(\overline{\mathbf{Q}}, \overline{\mathbf{T}}, \mathbf{C})\rangle\right] \\
= & \mathbb{E}\left[\langle\mathbf{d}, \overline{\mathbf{Q}}\rangle\left\langle\mathbf{d}, \mathbf{C} \cdot \mathbf{S}^{*}(\overline{\mathbf{Q}}, \overline{\mathbf{T}}, \mathbf{C})-\mathbf{A}\right\rangle\right],
\end{aligned}
$$

which is derived by setting the expected drift of $\langle\mathbf{d}, \mathbf{Q}\rangle^{2}$ to 0 .

Next, we give upper bounds for each individual term in the Left Hand Side (LHS) of (15) and a lower bound for the Right Hand Side (RHS) of (15). By simply setting the expected drift of $\langle\mathbf{d}, \mathbf{Q}\rangle$ equal to zero, we have

$$
\begin{aligned}
& \mathbb{E}[\langle\mathbf{d}, \mathbf{U}(\overline{\mathbf{Q}}, \overline{\mathbf{T}}, \mathbf{C})\rangle]=\left\langle\mathbf{d}, \mathbb{E}\left[\mathbf{C} \cdot \mathbf{S}^{*}(\overline{\mathbf{Q}}, \overline{\mathbf{T}}, \mathbf{C})\right]\right\rangle-\langle\mathbf{d}, \boldsymbol{\lambda}\rangle \\
& \stackrel{(a)}{=}\left\langle\mathbf{d}, \mathbb{E}\left[\mathbf{C} \cdot \mathbf{S}^{*}(\overline{\mathbf{Q}}, \overline{\mathbf{T}}, \mathbf{C})\right]\right\rangle-(b-\epsilon) \stackrel{(b)}{\leq} \epsilon,
\end{aligned}
$$

where the step (a) follows from the definition of $\boldsymbol{\lambda}^{(0)} \triangleq \boldsymbol{\lambda}+\epsilon \mathbf{d}$ and $\left\langle\mathbf{d}, \boldsymbol{\lambda}^{(0)}\right\rangle=b$ ( see Fig. 2); (b) follows from the facts that $\mathbb{E}\left[\mathbf{C} \cdot \mathbf{S}^{*}(\overline{\mathbf{Q}}, \overline{\mathbf{T}}, \mathbf{C})\right]$ must be within capacity region $\mathcal{R}$ and that $\langle\mathbf{d}, \mathbf{r}\rangle \leq b$ for any vector $\mathbf{r} \in \mathcal{R}$.

We are ready to provide an upper bound on the first term in the LHS of (15). Noting the fact that the amount of unused service in one time slot at each link $l$ cannot be greater than the maximum channel rate $C_{\max }$, i.e., $U_{l} \leq C_{\max }$, we have

$$
\begin{aligned}
& \frac{1}{2} \mathbb{E}\left[\langle\mathbf{d}, \mathbf{U}(\overline{\mathbf{Q}}, \overline{\mathbf{T}}, \mathbf{C})\rangle^{2}\right] \\
\leq & \frac{1}{2}\left\langle\mathbf{d}, C_{\max } \mathbf{1}\right\rangle \mathbb{E}[\langle\mathbf{d}, \mathbf{U}(\overline{\mathbf{Q}}, \overline{\mathbf{T}}, \mathbf{C})\rangle] \\
\leq & \frac{\epsilon}{2}\left\langle\mathbf{d}, C_{\max } \mathbf{1}\right\rangle,
\end{aligned}
$$

where the last step utilizes inequality (16).

Next, we focus on the second term in the LHS of (15). The system stability under the RSG Algorithm implies that it selects the schedule $\mathbf{S}$ which maximizes $\langle\mathbf{d}, \mathbf{S}\rangle$ with high probability when the arrival rate vector is very close to the face $\mathcal{H}^{(\mathrm{d})}$. See Fig. 6a for an example. Based on this observation, we can show

$$
\begin{aligned}
& \mathbb{E}\left[\left\langle\mathbf{d}, \mathbf{A}-\mathbf{C} \cdot \mathbf{S}^{*}(\overline{\mathbf{Q}}, \overline{\mathbf{T}}, \mathbf{C})\right\rangle^{2}\right] \\
& \leq \zeta^{(\epsilon)}+\sum_{\mathbf{c} \in \mathcal{C}} \frac{\epsilon}{\chi_{\mathbf{c}}}\left(2 b b_{\mathbf{c}}+\left(b_{\mathbf{c}}\right)^{2}+\left\langle\mathbf{d}, C_{\max } \mathbf{1}\right\rangle^{2}\right),
\end{aligned}
$$

where we recall that $\zeta^{(\epsilon)} \triangleq\left\langle\mathbf{d}^{2},\left(\boldsymbol{\sigma}^{(\epsilon)}\right)^{2}\right\rangle+\operatorname{Var}(\Psi)+\epsilon^{2}$ is defined in Proposition 2, and

$\chi_{\mathbf{c}} \triangleq \min \left\{b_{\mathbf{c}}-\langle\mathbf{d}, \mathbf{r}\rangle:\right.$ for all $\left.\mathbf{r} \in \mathcal{S}^{(\mathbf{c})} \backslash\left\{\mathbf{w}: b_{\mathbf{c}}=\langle\mathbf{d}, \mathbf{w}\rangle\right\}\right\}$.

The detailed proof is provided in Appendix G. Inequality (18) indicates that the second moment of $\mathbf{d}$-weighted difference between arrivals and services is dominated by the $\mathbf{d}^{2}$-weighted variance of the arrival process and the variance of the channel fading process in the heavy-traffic limit.

In order to analyze the third term in the LHS of (15), we restrict vectors to $\left|\mathcal{L}_{+}\right|$-dimensional space, where $\mathcal{L}_{+} \triangleq\{l \in$ 


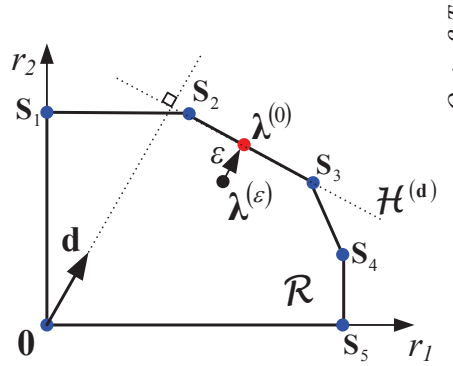

(a)

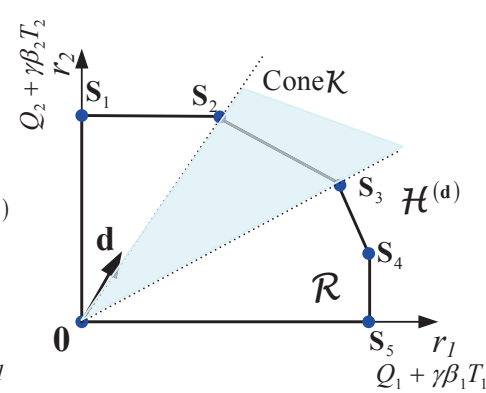

(b)
Fig. 6: Key properties of the RSG Algorithm (illustrated in non-fading case for an easier exposition): (a) when the arrival rate vector $\lambda^{(\epsilon)}$ is very close to the face $\mathcal{H}^{(\mathbf{d})}$, the RSG Algorithm should select the feasible schedules $\mathbf{S}_{2}$ and $\mathbf{S}_{3}$ with high probability due to the system stability; (b) when the vector $\mathbf{Q}+\gamma \boldsymbol{\beta} \cdot \mathbf{T}$ is within the cone $\mathcal{K}$, then the RSG Algorithm selects the schedules $\mathbf{S}_{2}$ and $\mathbf{S}_{3}$.

$\left.\mathcal{L}: d_{l}>0\right\}$. We use $\widetilde{I} \triangleq\left(I_{l}\right)_{l \in \mathcal{L}_{+}}$to denote the vector $\mathbf{I}$ restricted in the $\left|\mathcal{L}_{+}\right|$-dimensional space. Therefore, we have

$$
\begin{aligned}
& \mathbb{E}\left[\left\langle\mathbf{d}, \mathbf{Q}[t]+A[t]-\mathbf{C}[t] \cdot \mathbf{S}^{*}[t]\right\rangle\langle\mathbf{d}, \mathbf{U}[t]\rangle\right] \\
& \stackrel{(a)}{=} \mathbb{E}[\langle\mathbf{d}, \mathbf{Q}[t+1]\rangle\langle\mathbf{d}, \mathbf{U}[t]\rangle]-\mathbb{E}\left[\langle\mathbf{d}, \mathbf{U}[t]\rangle^{2}\right] \\
& \stackrel{(b)}{\leq} \mathbb{E}[\langle\mathbf{d}, \mathbf{Q}[t+1]\rangle\langle\mathbf{d}, \mathbf{U}[t]\rangle] \\
&= \mathbb{E}[\langle\widetilde{\mathbf{d}}, \widetilde{\mathbf{Q}}[t+1]\rangle\langle\widetilde{\mathbf{d}}, \widetilde{\mathbf{U}}[t]\rangle] \\
& \stackrel{(c)}{=} \mathbb{E}\left[\left\langle-\widetilde{\mathbf{Q}}_{\perp}[t+1], \widetilde{\mathbf{U}}[t]\right\rangle\right] \\
& \stackrel{(d)}{\leq} \sqrt{\mathbb{E}\left[\left\|\widetilde{\mathbf{Q}}_{\perp}[t+1]\right\|^{2}\right] \mathbb{E}\left[\|\widetilde{\mathbf{U}}[t]\|^{2}\right]} \\
& \stackrel{(e)}{\leq} \sqrt{\mathbb{E}\left[\left\|\mathbf{Q}_{\perp}[t+1]\right\|^{2}\right] \frac{C_{\max }}{d_{\min }} \mathbb{E}[\langle\mathbf{d}, \mathbf{U}[t]\rangle]},
\end{aligned}
$$

where the step $(a)$ utilizes equation $(1) ;(b)$ is true since $\mathbb{E}\left[\langle\mathbf{d}, \mathbf{U}[t]\rangle^{2}\right] \geq 0 ;(c)$ utilizes [4, Lemma 9]; $(d)$ uses CauchySchwarz inequality; $(e)$ follows from the fact that $\|\widetilde{\mathbf{I}}\| \leq\|\mathbf{I}\|$ for any vector $\mathbf{I}$ and the fact that $\widetilde{U}_{l} \leq C_{\max }, \forall l \in$ $\mathcal{L}_{+}$and $d_{\text {min }} \triangleq \min _{l \in \mathcal{L}_{+}} d_{l}$. By taking the limit $t \rightarrow \infty$ on both sides of (19), we have

$$
\begin{aligned}
& \mathbb{E}\left[\left\langle\mathbf{d}, \overline{\mathbf{Q}}+\mathbf{A}-\mathbf{C} \cdot \mathbf{S}^{*}(\overline{\mathbf{Q}}, \overline{\mathbf{T}}, \mathbf{C})\right\rangle\langle\mathbf{d}, \mathbf{U}(\overline{\mathbf{Q}}, \overline{\mathbf{T}}, \mathbf{C})\rangle\right] \\
\leq & \sqrt{\mathbb{E}\left[\left\|\overline{\mathbf{Q}}_{\perp}\right\|^{2}\right] \frac{C_{\max }}{d_{\min }} \mathbb{E}[\langle\mathbf{d}, \mathbf{U}(\overline{\mathbf{Q}}, \overline{\mathbf{T}}, \mathbf{C})\rangle]} \\
\leq & \sqrt{\epsilon \mathbb{E}\left[\left\|\overline{\mathbf{Q}}_{\perp}\right\|^{2}\right] \frac{C_{\max }}{d_{\min }}}
\end{aligned}
$$

where the last step uses inequality (16).

Finally, we consider the RHS of (15). By using the definition of the RSG Algorithm, we can show

$$
\begin{aligned}
& \mathbb{E}\left[\langle\mathbf{d}, \overline{\mathbf{Q}}\rangle\left\langle\mathbf{d}, \mathbf{C} \cdot \mathbf{S}^{*}(\overline{\mathbf{Q}}, \overline{\mathbf{T}}, \mathbf{C})-\mathbf{A}\right\rangle\right] \\
\geq & \epsilon \mathbb{E}\left[\left\|\overline{\mathbf{Q}}_{\|}\right\|\right]-\cot (\theta) \sqrt{\left(\mathbb{E}\left[\left\|\overline{\mathbf{Q}}_{\perp}\right\|^{2}\right]+(\gamma(\epsilon))^{2} N_{\mathbf{T}, 2}\right) \epsilon} \\
& \times \sqrt{\sum_{\mathbf{c} \in \mathcal{C}} \frac{1}{\chi_{\mathbf{c}}}\left(\left(b_{\mathbf{c}}\right)^{2}+\left\langle\mathbf{d}, C_{\max } \mathbf{1}\right\rangle^{2}\right)},
\end{aligned}
$$

where $\theta \in\left(0, \frac{\pi}{2}\right]$ is an angle such that $\left\langle\mathbf{d}, \mathbf{R}^{*}(\mathbf{Q}, \mathbf{T})\right\rangle=b$, for all $\mathbf{Q}$ and $\mathbf{T}$ satisfying $\frac{\left\|(\mathbf{Q}+\gamma \boldsymbol{\beta} \cdot \mathbf{T})_{\|}\right\|}{\|\mathbf{Q}+\gamma \boldsymbol{\beta} \cdot \mathbf{T}\|} \geq \cos (\theta), \mathbf{R}^{*}(\mathbf{Q}, \mathbf{T}) \triangleq$ $\mathbb{E}\left[\mathbf{C} \cdot \mathbf{S}^{*}(\mathbf{Q}, \mathbf{T}, \mathbf{C}) \mid \mathbf{Q}, \mathbf{T}\right]$ (Fig. 6b provides an example illustrating this fact), and $N_{\mathbf{T}, 2}$ (cf. Proposition 3) is independent of $\epsilon$. The proof is provided in Appendix H.

By substituting bounds (17), (18), (20) and (21) into identity (15), we have

$$
\epsilon \mathbb{E}\left[\left\|\overline{\mathbf{Q}}_{\|}\right\|\right] \leq \frac{\zeta^{(\epsilon)}}{2}+\bar{B}^{(\epsilon)}
$$

$$
\text { where } \begin{aligned}
\bar{B}^{(\epsilon) \triangleq} & \frac{\epsilon}{2}\left\langle\mathbf{d}, C_{\max } \mathbf{1}\right\rangle+\sqrt{\epsilon \mathbb{E}\left[\left\|\overline{\mathbf{Q}}_{\perp}\right\|^{2}\right] \frac{C_{\max }}{c_{\min }}} \\
& +\frac{1}{2} \sum_{\mathbf{c} \in \mathcal{C}} \frac{\epsilon}{\chi_{\mathbf{c}}}\left(2 b b_{\mathbf{c}}+\left(b_{\mathbf{c}}\right)^{2}+\left\langle\mathbf{d}, C_{\max } \mathbf{1}\right\rangle^{2}\right) \\
& +\cot (\theta) \sqrt{2\left(\mathbb{E}\left[\left\|\overline{\mathbf{Q}}_{\perp}\right\|^{2}\right]+(\gamma(\epsilon))^{2} N_{\mathbf{T}, 2}\right) \epsilon} \\
& \times \sqrt{\sum_{\mathbf{c} \in \mathcal{C}} \frac{1}{\chi_{\mathbf{c}}}\left(\left(b_{\mathbf{c}}\right)^{2}+\left\langle\mathbf{d}, C_{\max } \mathbf{1}\right\rangle^{2}\right)} .
\end{aligned}
$$

Thus, if $\lim _{\epsilon \downarrow 0} \bar{B}^{(\epsilon)}=0$, then the RSG Algorithm is heavytraffic optimal by Definition 1 . Noting that $N_{\mathbf{T}, 2}$ is independent of $\epsilon$, to satisfy $\lim _{\epsilon \downarrow 0} \bar{B}^{(\epsilon)}=0$, it is sufficient to have

$$
\lim _{\epsilon \downarrow 0} \epsilon \mathbb{E}\left[\left\|\overline{\mathbf{Q}}_{\perp}\right\|^{2}\right]=0 \text { and } \lim _{\epsilon \downarrow 0} \epsilon(\gamma(\epsilon))^{2}=0 .
$$

By using the state-space collapse result established by Proposition 3 , it is easy to see that $\gamma(\epsilon)=O\left(\frac{1}{\sqrt[5]{\epsilon}}\right)$ meets the above requirements.

\section{CONCLUSION}

We studied the heavy-traffic behavior of the recently proposed maximum-weight type scheduling algorithm, called Regular Service Guarantee (RSG) Algorithm, where each link weight consists of its own queue-length and a counter, namely the time-since-last-service (TSLS), which tracks the time since the last service. The RSG Algorithm has been shown to achieve throughput optimality while providing regular service guarantees. In this paper, we further showed that the RSG Algorithm is heavy-traffic optimal as long as its design parameter weighting for the TSLS $\gamma=O\left(\frac{1}{\sqrt[5]{\epsilon}}\right)$, where $\epsilon$ is the heavytraffic parameter characterizing the closeness of the arrival rate vector to the boundary of the capacity region. Noting that the service regularity improves with increasing $\gamma$, our result reveals that the RSG Algorithm with a carefully selected parameter $\gamma$ can achieve the best service regularity performance among the class of the RSG Algorithms without sacrificing the mean delay optimality under heavy-traffic conditions.

\section{APPENDIX A}

\section{PROOF OF LEMMA 1}

We will omit $\epsilon$ associated with queue length processes, TSLS counters and parameter $\gamma(\epsilon)$ for brevity. Also, we will use $\mathbf{I}^{+}$to denote $\mathbf{I}[t+1]$ for any vector $\mathbf{I}$ in the rest of proof. 
First, we note that

$$
\begin{aligned}
& \Delta V_{\perp}(\mathbf{Q}, \mathbf{T}) \triangleq V_{\perp}\left(\mathbf{Q}^{+}, \mathbf{T}^{+}\right)-V_{\perp}(\mathbf{Q}, \mathbf{T}) \\
= & \sqrt{\left\|\left(\mathbf{Q}_{\perp}^{+}, \sqrt{2 \gamma C_{\max } \boldsymbol{\beta} \cdot \mathbf{T}^{+}}\right)\right\|^{2}}-\sqrt{\left\|\left(\mathbf{Q}_{\perp}, \sqrt{2 \gamma C_{\max } \boldsymbol{\beta} \cdot \mathbf{T}}\right)\right\|^{2}} \\
\stackrel{(a)}{\leq} & \frac{\left\|\left(\mathbf{Q}_{\perp}^{+}, \sqrt{2 \gamma C_{\max } \boldsymbol{\beta} \cdot \mathbf{T}^{+}}\right)\right\|^{2}-\left\|\left(\mathbf{Q}_{\perp}, \sqrt{2 \gamma C_{\max } \boldsymbol{\beta} \cdot \mathbf{T}}\right)\right\|^{2}}{2\left\|\left(\mathbf{Q}_{\perp}, \sqrt{2 \gamma C_{\max } \boldsymbol{\beta} \cdot \mathbf{T}}\right)\right\|} \\
\stackrel{(b)}{=} & \frac{\left(W\left(\mathbf{Q}^{+}, \mathbf{T}^{+}\right)-W(\mathbf{Q}, \mathbf{T})\right)-\left(W_{\|}\left(\mathbf{Q}^{+}, \mathbf{T}^{+}\right)-W_{\|}(\mathbf{Q}, \mathbf{T})\right)}{2\left\|\left(\mathbf{Q}_{\perp}, \sqrt{2 \gamma C_{\max } \boldsymbol{\beta} \cdot \mathbf{T}}\right)\right\|} \\
\stackrel{(c)}{=} & \frac{\Delta W(\mathbf{Q}, \mathbf{T})-\Delta W_{\|}(\mathbf{Q}, \mathbf{T})}{2\left\|\left(\mathbf{Q}_{\perp}, \sqrt{2 \gamma C_{\max } \boldsymbol{\beta} \cdot \mathbf{T}}\right)\right\|},
\end{aligned}
$$

where the step $(a)$ follows from the fact that $f(x)=$ $\sqrt{x}$ for $x \geq 0$ so that $f(y)-f(x) \leq f^{\prime}(x)(y-$ $x)=\frac{y-x}{2 \sqrt{x}}$ with $y=\left\|\left(\mathbf{Q}_{\perp}^{+}, \sqrt{2 \gamma C_{\max } \boldsymbol{\beta} \cdot \mathbf{T}^{+}}\right)\right\|^{2}$ and $x=$ $\left\|\left(\mathbf{Q}_{\perp}, \sqrt{2 \gamma C_{\max } \boldsymbol{\beta} \cdot \mathbf{T}}\right)\right\|^{2} ;(b)$ is true since $\|(\mathbf{I}, \mathbf{J})\|^{2}=\|\mathbf{I}\|^{2}+$ $\|\mathbf{J}\|^{2}$ for any vectors $\mathbf{I}, \mathbf{J},\|\mathbf{I}\|^{2}=\left\|\mathbf{I}_{\|}\right\|^{2}+\left\|\mathbf{I}_{\perp}\right\|^{2}, W(\mathbf{Q}, \mathbf{T}) \triangleq$ $\left\|\left(\mathbf{Q}, \sqrt{2 \gamma C_{\max } \boldsymbol{\beta} \cdot \mathbf{T}}\right)\right\|^{2}$, and $W_{\|}(\mathbf{Q}, \mathbf{T}) \triangleq\left\|\mathbf{Q}_{\|}\right\|^{2} ;(c)$ is true by letting

$$
\begin{aligned}
& \Delta W(\mathbf{Q}, \mathbf{T}) \triangleq W\left(\mathbf{Q}^{+}, \mathbf{T}^{+}\right)-W(\mathbf{Q}, \mathbf{T}), \\
& \Delta W_{\|}(\mathbf{Q}, \mathbf{T}) \triangleq W_{\|}\left(\mathbf{Q}^{+}, \mathbf{T}^{+}\right)-W_{\|}(\mathbf{Q}, \mathbf{T}) .
\end{aligned}
$$

Having inequality (25), we can first study the conditional expectation of $\Delta W(\mathbf{Q}, \mathbf{T})$ and $\Delta W_{\|}(\mathbf{Q}, \mathbf{T})$ instead of directly studying the conditional expectation of $\Delta V_{\perp}(\mathbf{Q}, \mathbf{T})$. We first focus on $\mathbb{E}[\Delta W(\mathbf{Q}, \mathbf{T}) \mid \mathbf{Q}, \mathbf{T}]$.

$$
\begin{aligned}
& \mathbb{E}[\Delta W(\mathbf{Q}, \mathbf{T}) \mid \mathbf{Q}, \mathbf{T}] \\
& =\mathbb{E}\left[\left\|\left(\mathbf{Q}^{+}, \sqrt{2 \gamma C_{\max } \boldsymbol{\beta} \cdot \mathbf{T}^{+}}\right)\right\|^{2}\right. \\
& \left.-\left\|\left(\mathbf{Q}, \sqrt{2 \gamma C_{\max } \boldsymbol{\beta} \cdot \mathbf{T}}\right)\right\|^{2} \mid \mathbf{Q}, \mathbf{T}\right] \\
& =\mathbb{E}\left[\left\|\mathbf{Q}^{+}\right\|^{2}+2 \gamma C_{\max }\left\|\boldsymbol{\beta} \cdot \mathbf{T}^{+}\right\|_{1}\right. \\
& \left.-\|\mathbf{Q}\|^{2}-2 \gamma C_{\max }\|\boldsymbol{\beta} \cdot \mathbf{T}\|_{1} \mid \mathbf{Q}, \mathbf{T}\right] \\
& =\mathbb{E}\left[\left\|\mathbf{Q}+\mathbf{A}-\mathbf{S}^{*} \cdot \mathbf{C}+\mathbf{U}\right\|^{2}-\|\mathbf{Q}\|^{2}\right. \\
& \left.+2 \gamma C_{\max }\left(\left\|\boldsymbol{\beta} \cdot \mathbf{T}^{+}\right\|_{1}-\|\boldsymbol{\beta} \cdot \mathbf{T}\|_{1}\right) \mid \mathbf{Q}, \mathbf{T}\right] \\
& =\mathbb{E}\left[\left\|\mathbf{Q}+\mathbf{A}-\mathbf{S}^{*} \cdot \mathbf{C}\right\|^{2}+2\left\langle\mathbf{Q}+\mathbf{A}-\mathbf{S}^{*} \cdot \mathbf{C}, \mathbf{U}\right\rangle+\|\mathbf{U}\|^{2}\right. \\
& \left.-\|\mathbf{Q}\|^{2}+2 \gamma C_{\max }\left(\left\|\boldsymbol{\beta} \cdot \mathbf{T}^{+}\right\|_{1}-\|\boldsymbol{\beta} \cdot \mathbf{T}\|_{1}\right) \mid \mathbf{Q}, \mathbf{T}\right] \\
& \stackrel{(a)}{\leq} \mathbb{E}\left[\left\|\mathbf{Q}+\mathbf{A}-\mathbf{S}^{*} \cdot \mathbf{C}\right\|^{2}-\|\mathbf{Q}\|^{2}\right. \\
& \left.+2 \gamma C_{\max }\left(\left\|\boldsymbol{\beta} \cdot \mathbf{T}^{+}\right\|_{1}-\|\boldsymbol{\beta} \cdot \mathbf{T}\|_{1}\right) \mid \mathbf{Q}, \mathbf{T}\right] \\
& \stackrel{(b)}{=} \mathbb{E}\left[2\left\langle\mathbf{Q}, \mathbf{A}-\mathbf{S}^{*} \cdot \mathbf{C}\right\rangle+\left\|\mathbf{A}-\mathbf{S}^{*} \cdot \mathbf{C}\right\|^{2}\right. \\
& \left.+2 \gamma C_{\max }\left(\sum_{l=1}^{L} \beta_{l}-\sum_{l \in \mathbf{H}^{*}} \beta_{l}-\sum_{l \in \mathbf{H}^{*}} \beta_{l} T_{l}\right) \mid \mathbf{Q}, \mathbf{T}\right]
\end{aligned}
$$

$$
\begin{aligned}
\stackrel{(c)}{\leq} 2 \mathbb{E}[ & \left.\left\langle\mathbf{Q}, \mathbf{A}-\mathbf{S}^{*} \cdot \mathbf{C}\right\rangle \mid \mathbf{Q}, \mathbf{T}\right]+K_{1} \\
& -2 \gamma \mathbb{E}\left[\left\langle\boldsymbol{\beta} \cdot \mathbf{T}, \mathbf{S}^{*} \cdot \mathbf{C}\right\rangle \mid \mathbf{Q}, \mathbf{T}\right],
\end{aligned}
$$

where the step $(a)$ uses the fact that $U_{l}[t]\left(Q_{l}[t]+A_{l}[t]-\right.$ $\left.S_{l}^{*}[t] C_{l}[t]\right)=-U_{l}^{2}[t]$ for each $l ;(b)$ follows from the fact that $\sum_{l=1}^{L} \beta_{l} T_{l}^{+}-\sum_{l=1}^{L} \beta_{l} T_{l}=\sum_{l=1}^{L} \beta_{l}-\sum_{l \in \mathbf{H}^{*}} \beta_{l}-\sum_{l \in \mathbf{H}^{*}} \beta_{l} T_{l}$ and $\mathbf{H}^{*} \triangleq\left\{l \in \mathcal{L}: S_{l}^{*} C_{l}>0\right\} ;(c)$ is true for $K_{1} \triangleq$ $L \max \left\{A_{\max }^{2}, C_{\max }^{2}\right\}+2 \gamma C_{\max } \sum_{l=1}^{L} \beta_{l}$.

Next, we consider $\mathbb{E}\left[\left\langle\mathbf{Q}, \mathbf{A}-\mathbf{S}^{*} \cdot \mathbf{C}\right\rangle \mid \mathbf{Q}, \mathbf{T}\right]$. By using the definition of projection $\boldsymbol{\lambda}^{(0)}$, we have

$$
\begin{aligned}
& \mathbb{E}\left[\left\langle\mathbf{Q}, \mathbf{A}-\mathbf{S}^{*} \cdot \mathbf{C}\right\rangle \mid \mathbf{Q}, \mathbf{T}\right] \\
= & \left\langle\mathbf{Q}, \boldsymbol{\lambda}^{(0)}-\epsilon \mathbf{d}\right\rangle-\mathbb{E}\left[\left\langle\mathbf{Q}, \mathbf{S}^{*} \cdot \mathbf{C}\right\rangle \mid \mathbf{Q}, \mathbf{T}\right] \\
=- & \epsilon\left\|\mathbf{Q}_{\|}\right\|+\left\langle\mathbf{Q}, \boldsymbol{\lambda}^{(0)}\right\rangle-\mathbb{E}\left[\left\langle\mathbf{Q}+\gamma \boldsymbol{\beta} \cdot \mathbf{T}, \mathbf{S}^{*} \cdot \mathbf{C}\right\rangle \mid \mathbf{Q}, \mathbf{T}\right] \\
& +\gamma \mathbb{E}\left[\left\langle\boldsymbol{\beta} \cdot \mathbf{T}, \mathbf{S}^{*} \cdot \mathbf{C}\right\rangle \mid \mathbf{Q}, \mathbf{T}\right] .
\end{aligned}
$$

Given the queue-length vector $\mathbf{Q}$ and TSLS vector $\mathbf{T}$ at the beginning of each slot, according to the definition of the RSG Algorithm and the capacity region $\mathcal{R}$, it is easy to see that

$$
\left\langle\mathbf{Q}+\gamma \boldsymbol{\beta} \cdot \mathbf{T}, \mathbb{E}\left[\mathbf{S}^{*} \cdot \mathbf{C} \mid \mathbf{Q}, \mathbf{T}\right]\right\rangle=\max _{\mathbf{r} \in \mathcal{R}}\langle\mathbf{Q}+\gamma \boldsymbol{\beta} \cdot \mathbf{T}, \mathbf{r}\rangle
$$

Since $\boldsymbol{\lambda}^{(0)}$ is a relative interior point of dominant hyperplane $\mathcal{H}^{(\mathbf{d})}$, there exists a small enough $\delta>0$ such that $\mathcal{B}_{\delta} \triangleq \mathcal{H}^{(\mathbf{d})} \bigcap\left\{\mathbf{r} \succ \mathbf{0}:\left\|\mathbf{r}-\boldsymbol{\lambda}^{(0)}\right\| \leq \delta\right\}$ representing the set of vectors on the hyperplane $\mathcal{H}^{(\mathbf{d})}$ that are within $\delta$ distance from $\lambda^{(0)}$, lies strictly within the face $\mathcal{F}^{(\mathbf{d})} \triangleq \mathcal{H}^{(\mathbf{d})} \cap \mathcal{R}$. Therefore, we have

$$
\begin{aligned}
\max _{\mathbf{r} \in \mathcal{R}}\langle\mathbf{Q}+\gamma \boldsymbol{\beta} \cdot \mathbf{T}, \mathbf{r}\rangle & \geq \max _{\mathbf{r} \in \mathcal{B}_{\delta}}\langle\mathbf{Q}+\gamma \boldsymbol{\beta} \cdot \mathbf{T}, \mathbf{r}\rangle \\
& \geq \max _{\mathbf{r} \in \mathcal{B}_{\delta}}\langle\mathbf{Q}, \mathbf{r}\rangle+\left\langle\gamma \boldsymbol{\beta} \cdot \mathbf{T}, \mathbf{r}^{*}\right\rangle,
\end{aligned}
$$

where $\mathbf{r}^{*} \in \underset{\mathbf{r} \in \mathcal{B}_{\delta}}{\arg \max }\langle\mathbf{Q}, \mathbf{r}\rangle$.

Since normal vector $\mathbf{d} \succeq \mathbf{0}$ and arrival rate vector $\boldsymbol{\lambda} \succ \mathbf{0}$, we have $\boldsymbol{\lambda}^{(0)} \succ 0$. Therefore, we can find a $\delta>0$ sufficiently small such that $r_{l} \geq r_{\min }$ for all $\mathbf{r}=\left(r_{l}\right)_{l \in \mathcal{L}} \in \mathcal{B}_{\delta}$ and some $r_{\min }>0$. Hence, by (28) and (29), we have

$\left\langle\mathbf{Q}+\gamma \boldsymbol{\beta} \cdot \mathbf{T}, \mathbb{E}\left[\mathbf{S}^{*} \cdot \mathbf{C} \mid \mathbf{Q}, \mathbf{T}\right]\right\rangle \geq \max _{\mathbf{r} \in \mathcal{B}_{\delta}}\langle\mathbf{Q}, \mathbf{r}\rangle+\gamma r_{\min }\|\boldsymbol{\beta} \cdot \mathbf{T}\|_{1}$.

By substituting above inequality into (27), we have

$$
\begin{aligned}
\mathbb{E}[ & \left.\left\langle\mathbf{Q}, \mathbf{A}-\mathbf{S}^{*} \cdot \mathbf{C}\right\rangle \mid \mathbf{Q}, \mathbf{T}\right] \\
\leq- & \epsilon\left\|\mathbf{Q}_{\|}\right\|+\min _{\mathbf{r} \in \mathcal{B}_{\delta}}\left\langle\mathbf{Q}, \boldsymbol{\lambda}^{(0)}-\mathbf{r}\right\rangle \\
& -\gamma r_{\min }\|\boldsymbol{\beta} \cdot \mathbf{T}\|_{1}+\gamma \mathbb{E}\left[\left\langle\boldsymbol{\beta} \cdot \mathbf{T}, \mathbf{S}^{*} \cdot \mathbf{C}\right\rangle \mid \mathbf{Q}, \mathbf{T}\right] .
\end{aligned}
$$

Since $\boldsymbol{\lambda}^{(0)}-\mathbf{r}$ is perpendicular to the normal vector $\mathbf{d}$ for $\mathbf{r} \in \mathcal{B}_{\delta}$, we have

$$
\min _{\mathbf{r} \in \mathcal{B}_{\delta}}\left\langle\mathbf{Q}, \boldsymbol{\lambda}^{(0)}-\mathbf{r}\right\rangle=\min _{\mathbf{r} \in \mathcal{B}_{\delta}}\left\langle\mathbf{Q}_{\perp}, \boldsymbol{\lambda}^{(0)}-\mathbf{r}\right\rangle=-\delta\left\|\mathbf{Q}_{\perp}\right\| .
$$

Hence, we have

$$
\begin{aligned}
& \mathbb{E}\left[\left\langle\mathbf{Q}, \mathbf{A}-\mathbf{S}^{*} \cdot \mathbf{C}\right\rangle \mid \mathbf{Q}, \mathbf{T}\right] \leq-\epsilon\left\|\mathbf{Q}_{\|}\right\|-\delta\left\|\mathbf{Q}_{\perp}\right\| \\
& \quad-\gamma r_{\min }\|\boldsymbol{\beta} \cdot \mathbf{T}\|_{1}+\gamma \mathbb{E}\left[\left\langle\boldsymbol{\beta} \cdot \mathbf{T}, \mathbf{S}^{*} \cdot \mathbf{C}\right\rangle \mid \mathbf{Q}, \mathbf{T}\right]
\end{aligned}
$$

Thus, by substituting (30) into (26), we have

$$
\begin{aligned}
\mathbb{E}[\Delta W(\mathbf{Q}, \mathbf{T}) \mid \mathbf{Q}, \mathbf{T}] \leq & -2 \epsilon\left\|\mathbf{Q}_{\|}\right\|-2 \delta\left\|\mathbf{Q}_{\perp}\right\| \\
& -2 \gamma r_{\min }\|\boldsymbol{\beta} \cdot \mathbf{T}\|_{1}+K_{1} .
\end{aligned}
$$


Next, we lower bound $\mathbb{E}\left[\Delta W_{\|}(\mathbf{Q}, \mathbf{T}) \mid \mathbf{Q}, \mathbf{T}\right]$.

$$
\begin{aligned}
& \mathbb{E}\left[\Delta W_{\|}(\mathbf{Q}, \mathbf{T}) \mid \mathbf{Q}, \mathbf{T}\right] \\
= & \mathbb{E}\left[\left\langle\mathbf{d}, \mathbf{Q}^{+}\right\rangle^{2}-\langle\mathbf{d}, \mathbf{Q}\rangle^{2} \mid \mathbf{Q}, \mathbf{T}\right] \\
= & \mathbb{E}\left[\left\langle\mathbf{d}, \mathbf{Q}+\mathbf{A}-\mathbf{S}^{*} \cdot \mathbf{C}+\mathbf{U}\right\rangle^{2}-\langle\mathbf{d}, \mathbf{Q}\rangle^{2} \mid \mathbf{Q}, \mathbf{T}\right] \\
= & \mathbb{E}\left[\left\langle\mathbf{d}, \mathbf{Q}+\mathbf{A}-\mathbf{S}^{*} \cdot \mathbf{C}\right\rangle^{2}+\langle\mathbf{d}, \mathbf{U}\rangle^{2}\right. \\
& \left.+2\left\langle\mathbf{d}, \mathbf{Q}+\mathbf{A}-\mathbf{S}^{*} \cdot \mathbf{C}\right\rangle\langle\mathbf{d}, \mathbf{U}\rangle-\langle\mathbf{d}, \mathbf{Q}\rangle^{2} \mid \mathbf{Q}, \mathbf{T}\right] \\
= & \mathbb{E}\left[2\langle\mathbf{d}, \mathbf{Q}\rangle\left\langle\mathbf{d}, \mathbf{A}-\mathbf{S}^{*} \cdot \mathbf{C}\right\rangle+\left\langle\mathbf{d}, \mathbf{A}-\mathbf{S}^{*} \cdot \mathbf{C}\right\rangle^{2}\right. \\
& \left.+2\left\langle\mathbf{d}, \mathbf{Q}+\mathbf{A}-\mathbf{S}^{*} \cdot \mathbf{C}\right\rangle\langle\mathbf{d}, \mathbf{U}\rangle+\langle\mathbf{d}, \mathbf{U}\rangle^{2} \mid \mathbf{Q}, \mathbf{T}\right] \\
= & 2\langle\mathbf{d}, \mathbf{Q}\rangle\left\langle\mathbf{d}, \boldsymbol{\lambda}-\mathbb{E}\left[\mathbf{S}^{*} \cdot \mathbf{C} \mid \mathbf{Q}, \mathbf{T}\right]\right\rangle \\
& -2 \mathbb{E}\left[\left\langle\mathbf{d}, \mathbf{S}^{*} \cdot \mathbf{C}\right\rangle\langle\mathbf{d}, \mathbf{U}\rangle \mid \mathbf{Q}, \mathbf{T}\right]+\mathbb{E}\left[\langle\mathbf{d}, \mathbf{U}\rangle^{2} \mid \mathbf{Q}, \mathbf{T}\right] \\
& +\mathbb{E}\left[\left\langle\mathbf{d}, \mathbf{A}-\mathbf{S}^{*} \cdot \mathbf{C}\right\rangle^{2}+2\langle\mathbf{d}, \mathbf{Q}+\mathbf{A}\rangle\langle\mathbf{d}, \mathbf{U}\rangle \mid \mathbf{Q}, \mathbf{T}\right] \\
\geq & 2\langle\mathbf{d}, \mathbf{Q}\rangle\left\langle\mathbf{d}, \boldsymbol{\lambda}-\mathbb{E}\left[\mathbf{S}^{*} \cdot \mathbf{C} \mid \mathbf{Q}, \mathbf{T}\right]\right\rangle \\
& -2 \mathbb{E}\left[\left\langle\mathbf{d}, \mathbf{S}^{*} \cdot \mathbf{C}\right\rangle\langle\mathbf{d}, \mathbf{U}\rangle \mid \mathbf{Q}, \mathbf{T}\right] \\
(a) & 2\langle\mathbf{d}, \mathbf{Q}\rangle\left\langle\mathbf{d}, \boldsymbol{\lambda}-\mathbb{E}\left[\mathbf{S}^{*} \cdot \mathbf{C} \mid \mathbf{Q}, \mathbf{T}\right]\right\rangle-K_{2} \\
\stackrel{(b)}{=} & -2 \epsilon\|\mathbf{Q}\| \|-K_{2} \\
& +2\|\mathbf{Q}\| \|\left(\left\langle\mathbf{d}, \boldsymbol{\lambda}^{(0)}\right\rangle-\left\langle\mathbf{d}, \mathbb{E}\left[\mathbf{S}^{*} \cdot \mathbf{C} \mid \mathbf{Q}, \mathbf{T}\right]\right\rangle\right) \\
(c) & -2 \epsilon\|\mathbf{Q}\| \|-K_{2},
\end{aligned}
$$

where the step (a) is true for $K_{2} \triangleq 2 L C_{\max }^{2}$; step (b) uses the definition of projection $\boldsymbol{\lambda}^{(0)}$; step (c) follows from the fact that $\mathbb{E}\left[\mathbf{S}^{*} \cdot \mathbf{C} \mid \mathbf{Q}, \mathbf{T}\right] \in \mathcal{R}, \mathcal{R} \subset\{\mathbf{r} \succeq \mathbf{0}:\langle\mathbf{d}, \mathbf{r}\rangle \leq b\}$ and $b=\left\langle\mathbf{d}, \boldsymbol{\lambda}^{(0)}\right\rangle$.

By using (31), (32) and (25), we have

$$
\begin{aligned}
& \mathbb{E}\left[\Delta V_{\perp}(\mathbf{Q}, \mathbf{T}) \mid \mathbf{Q}, \mathbf{T}\right] \\
\leq & \frac{\mathbb{E}\left[\Delta W(\mathbf{Q}, \mathbf{T})-\Delta W_{\|}(\mathbf{Q}, \mathbf{T}) \mid \mathbf{Q}, \mathbf{T}\right]}{2\left\|\left(\mathbf{Q}_{\perp}, \sqrt{2 \gamma C_{\max } \boldsymbol{\beta} \cdot \mathbf{T}}\right)\right\|} \\
\leq & \frac{-2 \delta\left\|\mathbf{Q}_{\perp}\right\|-2 \gamma r_{\min } \sum_{l=1}^{L} \beta_{l} T_{l}+K_{1}+K_{2}}{2\left\|\left(\mathbf{Q}_{\perp}, \sqrt{2 \gamma C_{\max } \boldsymbol{\beta} \cdot \mathbf{T}}\right)\right\|} .
\end{aligned}
$$

Note that $\gamma \beta_{l} T_{l} \geq \gamma \beta_{l} T_{l} \mathbb{1}_{\left\{\alpha T_{l} \geq 1\right\}} \geq \sqrt{\gamma \beta_{l} T_{l}} \mathbb{1}_{\left\{\gamma \beta_{l} T_{l} \geq 1\right\}}=$ $\sqrt{\gamma \beta_{l} T_{l}}-\sqrt{\gamma \beta_{l} T_{l}} \mathbb{1}_{\left\{\gamma \beta_{l} T_{l}<1\right\}} \geq \sqrt{\gamma \beta_{l} T_{l}}-1$, and $\left\|\mathbf{Q}_{\perp}\right\| \geq$ $\frac{1}{\sqrt{L}}\left\|\mathbf{Q}_{\perp}\right\|_{1}$, where $\mathbb{1}_{\{\cdot\}}$ is an indicator function. Thus, we have

$$
\begin{aligned}
& \mathbb{E}\left[\Delta V_{\perp}(\mathbf{Q}, \mathbf{T}) \mid \mathbf{Q}, \mathbf{T}\right] \\
\leq & \frac{-\frac{2 \delta}{\sqrt{L}}\left\|\mathbf{Q}_{\perp}\right\|_{1}-2 r_{\min } \sum_{l=1}^{L} \sqrt{\gamma \beta_{l} T_{l}}+K_{1}+K_{2}+2 L r_{\text {min }}}{2\left\|\left(\mathbf{Q}_{\perp}, \sqrt{2 \gamma C_{\max } \boldsymbol{\beta} \cdot \mathbf{T}}\right)\right\|} \\
\leq & -\min \left\{\frac{2 \delta}{\sqrt{L}}, \frac{\sqrt{2} r_{\min }}{\sqrt{C_{\max }}}\right\} \frac{\left\|\left(\mathbf{Q}_{\perp}, \sqrt{2 \gamma C_{\max } \boldsymbol{\beta} \cdot \mathbf{T}}\right)\right\|_{1}}{2\left\|\left(\mathbf{Q}_{\perp}, \sqrt{2 \gamma C_{\max } \boldsymbol{\beta} \cdot \mathbf{T}}\right)\right\|} \\
& +\frac{K_{1}+K_{2}+2 L r_{\min }}{2\left\|\left(\mathbf{Q}_{\perp}, \sqrt{2 \gamma C_{\max } \boldsymbol{\beta} \cdot \mathbf{T}}\right)\right\|} \\
\leq & -\min \left\{\frac{\delta}{\sqrt{L}}, \frac{r_{\min }}{\sqrt{2 C_{\max }}}\right\}+\frac{K_{1}+K_{2}+2 L r_{\min }}{2\left\|\left(\mathbf{Q}_{\perp}, \sqrt{2 \gamma C_{\max } \boldsymbol{\beta} \cdot \mathbf{T}}\right)\right\|} .
\end{aligned}
$$

where the last step uses the fact that $\|\mathbf{x}\|_{1} \geq\|\mathbf{x}\|$ for any vector $\mathbf{x}$. Hence, for any $0<\varsigma<\min \left\{\frac{\delta}{\sqrt{L}}, \frac{r_{\min }}{\sqrt{2 C_{\max }}}\right\}$, by taking

$$
\kappa \triangleq \frac{K_{1}+K_{2}+2 L r_{\min }}{2\left(\min \left\{\frac{\delta}{\sqrt{L}}, \frac{r_{\min }}{\sqrt{2 C_{\max }}}\right\}-\varsigma\right)},
$$

we have the desired result.

\section{APPENDIX B}

ProOF OF LEMMA 2

If the event

$$
\mathcal{E}_{l}[j] \triangleq\left\{C_{l}[j]>0, C_{i}[j]=0, \forall i \neq l ; A_{l}[j-1]>0\right\}
$$

happens for some $j \in[t-m+1, t)$, then under the RSG Algorithm, link $l$ should be scheduled at least once during the past $m$ slots and thus $T_{l}^{(\epsilon)}[t]<m$. This implies

$$
\begin{aligned}
& \operatorname{Pr}\left\{T_{l}^{(\epsilon)}[t] \geq m\right\} \\
\leq & \operatorname{Pr}\left\{\mathcal{E}_{l}[j] \text { does not happen for all } j \in[t-m+1, t)\right\} \\
= & \vartheta_{l}^{m} .
\end{aligned}
$$

where $\vartheta_{l} \triangleq 1-\left(1-q_{l}\right)\left(1-p_{l}\right) \Pi_{i \neq l} p_{i}$, and $q_{l} \triangleq \operatorname{Pr}\left\{A_{l}[t]=\right.$ $0\}, \forall l \in \mathcal{L}$. Since $\lambda_{l}>0$, we have $q_{l}<1$ and thus $\vartheta_{l} \in(0,1)$. Thus, by taking $\vartheta \triangleq \max _{l \in \mathcal{L}} \vartheta_{l}$, we have the desired result.

\section{APPENDIX C \\ PROOF OF PROPOSITION 3}

In the rest of proof, we will omit $\epsilon$ associated with the queue length processes, the TSLS counters and parameter $\gamma(\epsilon)$ for brevity. As we pointed it out in Section VI, it is challenging to directly provide an upper bound on $\mathbb{E}\left[\left\|\overline{\mathbf{Q}}_{\perp}\right\|^{2}\right]$. Therefore, we will first upper-bound the moment generation function of $\left\|\overline{\mathbf{Q}}_{\perp}\right\|$, and then establish the relationship between the moments of a random variable and its moment generation function to upper-bound $\mathbb{E}\left[\left\|\overline{\mathbf{Q}}_{\perp}\right\|^{2}\right]$.

Lemma 3: If $\operatorname{Pr}\left\{C_{l}[t]=0\right\}>0, \forall l \in \mathcal{L}$, then, under the RSG Algorithm, there exists a $\eta>0$ satisfying $\vartheta e^{\eta F_{1}}<1$ and $e^{\eta G_{2}}-\eta\left(G_{2}+\varsigma\right)<1$ such that

$$
\mathbb{E}\left[e^{\eta\left\|\overline{\mathbf{Q}}_{\perp}\right\|}\right] \leq \frac{G}{1-\rho},
$$

where

$$
\begin{aligned}
G \triangleq & e^{\eta G_{1}}+\frac{L e^{\eta F_{2}}}{1-e^{\eta F_{1} \vartheta}}, \\
\rho \triangleq & e^{\eta G_{2}}-\eta\left(G_{2}+\varsigma\right) \in(0,1), \\
F_{1} \triangleq & L \sqrt{2 \gamma C_{\max } \sum_{l=1}^{L} \beta_{l}}+\sqrt{1+2 \gamma C_{\max } L \sum_{l=1}^{L} \beta_{l}}, \\
F_{2} \triangleq & \frac{2 \gamma C_{\max } \sum_{l=1}^{L} \beta_{l}}{\kappa}+2 L \max \left\{A_{\max }, C_{\max }\right\}, \\
G_{1}^{2} \triangleq & \kappa^{2}+2\left(A_{\max }+2 \sqrt{L} C_{\max }\right) \kappa \sqrt{L} \\
& +L\left(A_{\max }+2 \sqrt{L} C_{\max }\right)^{2}+2 \gamma C_{\max } \sum_{l=1}^{L} \beta_{l}, \\
G_{2} \triangleq & 2 L \max \left\{A_{\max }, C_{\max }\right\}+\frac{2 \gamma C_{\max } \sum_{l=1}^{L} \beta_{l}}{\kappa} \\
& +2 \gamma C_{\max } L \sum_{l=1}^{L} \beta_{l},
\end{aligned}
$$

$\vartheta$ is defined in Lemma 2 , $\varsigma$ and $\kappa$ are defined in (33).

In Lemma 1 , we have show that $V_{\perp}(\mathbf{Q}, \mathbf{T}) \triangleq$ $\left\|\left(\mathbf{Q}_{\perp}, \sqrt{2 \gamma C_{\max } \boldsymbol{\beta} \cdot \mathbf{T}}\right)\right\|$ has a negative drift whenever 
$V_{\perp}(\mathbf{Q}, \mathbf{T})$ is large enough. However, as we pointed it out in Section VI, we cannot directly provide an upper bound on the moment generation function of $V_{\perp}(\mathbf{Q}, \mathbf{T})$ by [7, Theorem 2.3], which requires either boundedness or the exponential tail of the Lyapunov drift. This is due to the abrupt dynamics of TSLS counters. We resolve this issue by exploiting the coupling between the queue-length processes and TSLS counters. Please see Appendix D for details.

Since we are interested in the scaling law of $\gamma$ to preserve heavy-traffic optimality under the RSG Algorithm, we will write an upper bound on the moment generation function of $\left\|\overline{\mathbf{Q}}_{\perp}\right\|$ as a function of $\gamma$ based on Lemma 3 .

First, it is easy to see that $\kappa=O(\gamma), G_{1}=O(\gamma), G_{2}=$ $O(\gamma), F_{1}=O(\sqrt{\gamma})$ and $F_{2}=O(1)$. Note that we need to choose a $\eta>0$ such that

$$
\begin{array}{r}
\vartheta e^{\eta F_{1}}<1 \\
e^{\eta G_{2}}-\eta\left(G_{2}+\varsigma\right)<1
\end{array}
$$

It is not hard to verify that

$$
0<\eta \leq \frac{1}{2} \min \left\{\frac{1}{F_{1}} \ln \frac{1}{\vartheta}, \frac{1}{G_{2}} \ln \frac{G_{2}+\varsigma}{G_{2}}\right\}
$$

satisfies above requirements. If $\gamma$ is large enough such that $\frac{\varsigma}{G_{2}}<1$ and $G_{2} \gg F_{1}$, then we have

$$
\frac{1}{G_{2}} \ln \frac{G_{2}+\varsigma}{G_{2}} \leq \frac{1}{F_{1}} \ln \frac{1}{\vartheta}
$$

Thus, we can take $\eta^{*} \triangleq \frac{1}{2 G_{2}} \ln \frac{G_{2}+\varsigma}{G_{2}}$ to meet the above requirements, and hence $\eta^{*}=O\left(\frac{1}{\gamma^{2}}\right)$.

Taking $\eta=\eta^{*}$ and noting that $\eta^{*}<\frac{1}{2 F_{1}} \ln \frac{1}{\vartheta}$, we have

$$
\begin{aligned}
& \frac{G}{1-\rho}=\frac{e^{\eta^{*} G_{1}}+\frac{L e^{\eta^{*} F_{2}}}{1-\vartheta e^{\eta^{*} F_{1}}}}{1-\left(e^{\eta^{*} G_{2}}-\eta^{*}\left(G_{2}+\varsigma\right)\right)} \\
& \leq \frac{e^{\eta^{*} G_{1}}+\frac{L e^{\eta^{*} F_{2}}}{1-\sqrt{\vartheta}}}{1-\left(e^{\eta^{*} G_{2}}-\eta^{*}\left(G_{2}+\varsigma\right)\right)} \\
&= \frac{e^{\eta^{*} G_{1}}+\frac{L e^{\eta^{*} F_{2}}}{1-\sqrt{\vartheta}}}{1-\left(1+\frac{\varsigma}{G_{2}}\right)^{\frac{1}{2}}+\frac{1}{2}\left(1+\frac{\varsigma}{G_{2}}\right) \ln \left(1+\frac{\varsigma}{G_{2}}\right)} \\
& \stackrel{(a)}{=} O\left(\frac{\varsigma}{1-\left(1+\frac{\varsigma}{2 G_{2}}\right)+\frac{1}{2}\left(1+\frac{\varsigma}{G_{2}}\right) \frac{\varsigma}{G_{2}}}\right)=O\left(\gamma^{2}\right),
\end{aligned}
$$

where the step $(a)$ uses $\eta^{*} G_{1}=O\left(\frac{1}{\gamma}\right)$ and $\eta^{*} F_{2}=O\left(\frac{1}{\gamma^{2}}\right)$. Thus, by Lemma 3, we have $\mathbb{E}\left[e^{\eta^{*}\left\|\overline{\mathbf{Q}}_{\perp}\right\|}\right]=O\left(\gamma^{2}\right)$.

Having obtained the upper bound on $\mathbb{E}\left[e^{\eta^{*}\left\|\overline{\mathbf{Q}}_{\perp}\right\|}\right]$, we need to establish the relationship between the moments of a random variable and its moment generation function to upper-bound $\mathbb{E}\left[\left\|\overline{\mathbf{Q}}_{\perp}\right\|^{2}\right]$, as shown in the following lemma.

Lemma 4: For a random variable $X$ with $\mathbb{E}\left[e^{\eta X}\right]<\infty$ for some $\eta>0$, we have

$$
\mathbb{E}\left[X^{n}\right] \leq \frac{1}{\eta^{n}}\left(\log \left(e^{n-1} \mathbb{E}\left[e^{\eta X}\right]\right)\right)^{n},
$$

for $n=1,2,3 \cdots$.

Please see the Appendix F for the proof of Lemma 4.
By taking $n=2$ in Lemma 4, we have the desired result.

\section{APPENDIX D}

PROOF OF LEMMA 3

Let $\mathbf{Z}[t] \triangleq\left(\mathbf{Q}_{\perp}[t], \sqrt{2 \gamma C_{\max } \boldsymbol{\beta} \cdot \mathbf{T}[t]}\right)$. We first give an upper bound on $\mathbb{E}\left[e^{\eta\|\mathbf{Z}[t+1]\|} \mid \mathbf{Q}[t], \mathbf{T}[t]\right]$. To that end, let $l^{*}[t] \in \arg \max _{l} \beta_{l} T_{l}[t]$. We partition $\left(\mathbf{Q}_{\perp}[t], \mathbf{T}[t]\right)$ into sets $\mathcal{F}_{1}, \mathcal{F}_{2}$ and $\mathcal{F}_{3}$, where

$\mathcal{F}_{1} \triangleq\{\|\mathbf{Z}[t]\| \leq \kappa\} ; \mathcal{F}_{2} \triangleq\left\{\|\mathbf{Z}[t]\|>\kappa,\left\|\mathbf{Q}_{\perp}[t]\right\|>T_{l^{*}[t]}[t]\right\} ;$ $\mathcal{F}_{3} \triangleq\left\{\|\mathbf{Z}[t]\|>\kappa,\left\|\mathbf{Q}_{\perp}[t]\right\| \leq T_{l^{*}[t]}[t]\right\}$.

Then, we have

$\mathbb{E}\left[e^{\eta\|\mathbf{Z}[t+1]\|} \mid \mathbf{Q}[t], \mathbf{T}[t]\right]=\sum_{i=1}^{3} \mathbb{E}\left[e^{\eta\|\mathbf{Z}[t+1]\|} ; \mathcal{F}_{i} \mid \mathbf{Q}[t], \mathbf{T}[t]\right]$.

Next, we consider each term in (47) individually.

(i) On event $\mathcal{F}_{1}$, we have

$$
\|\mathbf{Z}[t]\|=\sqrt{\left\|\mathbf{Q}_{\perp}[t]\right\|^{2}+2 \gamma C_{\max } \sum_{l=1}^{L} \beta_{l} T_{l}[t]} \leq \kappa,
$$

which implies $\left\|\mathbf{Q}_{\perp}[t]\right\| \leq \kappa$. For $\mathbf{Q}_{\perp}[t+1]$, we have

$$
\begin{aligned}
& \mathbf{Q}_{\perp}[t+1]=\mathbf{Q}[t+1]-\langle\mathbf{d}, \mathbf{Q}[t+1]\rangle \mathbf{d} \\
= & (\mathbf{Q}[t]+\mathbf{A}[t]-\mathbf{S}[t]+\mathbf{U}[t]) \\
& -\langle\mathbf{d}, \mathbf{Q}[t]+\mathbf{A}[t]-\mathbf{S}[t]+\mathbf{U}[t]\rangle \mathbf{d} \\
= & \mathbf{Q}[t]-\langle\mathbf{d}, \mathbf{Q}[t]\rangle \mathbf{d}+(\mathbf{A}[t]+\mathbf{U}[t]+\langle\mathbf{d}, \mathbf{S}[t]\rangle \mathbf{d}) \\
& -(\mathbf{S}[t]+\langle\mathbf{d}, \mathbf{A}[t]+\mathbf{U}[t]\rangle \mathbf{d}) \\
(a) & \mathbf{Q}_{\perp}[t]+A_{\max } \mathbf{1}+C_{\max } \mathbf{1}+\sqrt{L} C_{\max } \mathbf{d} \\
\preceq & \mathbf{Q}_{\perp}[t]+\left(A_{\max }+2 \sqrt{L} C_{\max }\right) \mathbf{1},
\end{aligned}
$$

where step $(a)$ uses the following inequality

$$
\langle\mathbf{d}, \mathbf{S}[t]\rangle \leq\|\mathbf{d}\|\|\mathbf{S}[t]\| \leq \sqrt{L} C_{\max }
$$

Hence, we have

$$
\begin{aligned}
& \quad\|\mathbf{Z}[t+1]\|^{2}=\left\|\mathbf{Q}_{\perp}[t+1]\right\|^{2}+2 \gamma C_{\max } \sum_{l=1}^{L} \beta_{l} T_{l}[t+1] \\
& \stackrel{(a)}{\leq}\left\|\mathbf{Q}_{\perp}[t]+\left(A_{\max }+2 \sqrt{L} C_{\max }\right) \mathbf{1}\right\|^{2} \\
& \quad+2 \gamma C_{\max } \sum_{l=1}^{L} \beta_{l}\left(T_{l}[t]+1\right) \\
& =\|\mathbf{Z}[t]\|^{2}+2\left(A_{\max }+2 \sqrt{L} C_{\max }\right)\left\|\mathbf{Q}_{\perp}[t]\right\|_{1} \\
& \quad+L\left(A_{\max }+2 \sqrt{L} C_{\max }\right)^{2}+2 \gamma C_{\max } \sum_{l=1}^{L} \beta_{l} \\
& \stackrel{(b)}{\leq} \kappa^{2}+2\left(A_{\max }+2 \sqrt{L} C_{\max }\right) \kappa \sqrt{L} \\
& +L\left(A_{\max }+2 \sqrt{L} C_{\max }\right)^{2}+2 \gamma C_{\max } \sum_{l=1}^{L} \beta_{l} \triangleq G_{1}^{2},
\end{aligned}
$$

where step (a) uses the inequality (48); step (b) utilizes the inequality $\|\mathbf{x}\|_{1} \leq \sqrt{L}\|\mathbf{x}\|$ for any $L$-dimensional vector $\mathbf{x}$. 
Hence, we have

$$
\mathbb{E}\left[e^{\eta\|\mathbf{Z}[t+1]\|} ; \mathcal{F}_{1} \mid \mathbf{Q}[t], \mathbf{T}[t]\right] \leq e^{\eta G_{1}}
$$

In order to analyze other two terms in (47), we need the following lemma.

Lemma 5: Under the RSG Algorithm, if $\|\mathbf{Z}[t]\|>\kappa$, then

$$
\begin{aligned}
& |\|\mathbf{Z}[t+1]\|-\|\mathbf{Z}[t]\|| \\
\leq & 2 L \max \left\{A_{\max }, C_{\max }\right\}+\frac{2 \gamma C_{\max } \sum_{l=1}^{L} \beta_{l}}{\kappa} \\
& +2 \gamma C_{\max } \frac{\sum_{l \in \mathbf{H}^{*}} \beta_{l} T_{l}[t]}{\sqrt{\left\|\mathbf{Q}_{\perp}[t]\right\|^{2}+2 \gamma C_{\max } \sum_{l=1}^{L} \beta_{l} T_{l}[t]}},
\end{aligned}
$$

where $\mathbf{H}^{*} \triangleq\left\{l: S_{l}^{*}[t] C_{l}[t]>0\right\}$.

The proof is available in Appendix E.

(ii) On event $\mathcal{F}_{2}$, we have

$$
\begin{aligned}
& \frac{\sum_{l \in \mathbf{H}^{*}} \beta_{l} T_{l}[t]}{\sqrt{\left\|\mathbf{Q}_{\perp}[t]\right\|^{2}+2 \gamma C_{\max } \sum_{l=1}^{L} \beta_{l} T_{l}[t]}} \\
\leq & \frac{L \beta_{l^{*}[t]} T_{l^{*}[t]}[t]}{\left\|\mathbf{Q}_{\perp}[t]\right\|} \leq L \sum_{l=1}^{L} \beta_{l} .
\end{aligned}
$$

By substituting above inequality into (51), we get

$$
|\|\mathbf{Z}[t+1]\|-\|\mathbf{Z}[t]\|| \leq G_{2}
$$

where $G_{2} \triangleq 2 L \max \left\{A_{\max }, C_{\max }\right\}+\frac{2 \gamma C_{\max } \sum_{l=1}^{L} \beta_{l}}{\kappa}+$ $2 \gamma C_{\max } L \sum_{l=1}^{L} \beta_{l}$. Noting that (12) and (52) satisfy conditions of [7, Lemma 2.2], there exists $\eta_{1}>0$ such that

$$
\mathbb{E}\left[e^{\eta(\|\mathbf{Z}[t+1]\|-\|\mathbf{Z}[t]\|)} ; \mathcal{F}_{2} \mid \mathbf{Q}[t], \mathbf{T}[t]\right] \leq \rho, \forall 0<\eta<\eta_{1},
$$

where $\rho \triangleq e^{\eta G_{2}}-\eta\left(G_{2}+\varsigma\right) \in(0,1)$, independent of $\epsilon$. Thus, we have

$$
\mathbb{E}\left[e^{\eta\|\mathbf{Z}[t+1]\|} ; \mathcal{F}_{2} \mid \mathbf{Q}[t], \mathbf{T}[t]\right] \leq \rho e^{\eta\|\mathbf{Z}[t]\|}
$$

(iii) On event $\mathcal{F}_{3}$, we have

$$
\begin{aligned}
& \frac{\sum_{l \in \mathbf{H}^{*}} \beta_{l} T_{l}[t]}{\sqrt{\left\|\mathbf{Q}_{\perp}[t]\right\|^{2}+2 \gamma C_{\max } \sum_{l=1}^{L} \beta_{l} T_{l}[t]}} \\
\leq & \frac{L \beta_{l^{*}[t]} T_{l^{*}[t]}[t]}{\sqrt{2 \gamma C_{\max } \beta_{l^{*}[t]} T_{l^{*}[t]}[t]}} \\
= & \frac{L}{\sqrt{2 \gamma C_{\max }}} \sqrt{\beta_{l^{*}[t]} T_{l^{*}[t]}[t]} \\
\leq & \frac{L \sqrt{\sum_{l=1}^{L} \beta_{l}}}{\sqrt{2 \gamma C_{\max }}} \sqrt{T_{l^{*}[t]}[t]} \\
\leq & \frac{L \sqrt{\sum_{l=1}^{L} \beta_{l}}}{\sqrt{2 \gamma C_{\max }}} T_{l^{*}[t]}[t] .
\end{aligned}
$$

By substituting (54) into (51), we get

$$
\begin{aligned}
& \|\mathbf{Z}[t+1]\|-\|\mathbf{Z}[t]\| \mid \\
\leq & 2 L \max \left\{A_{\max }, C_{\max }\right\}+\frac{2 \gamma C_{\max } \sum_{l=1}^{L} \beta_{l}}{\kappa} \\
& +T_{l^{*}[t]}[t] L \sqrt{2 \gamma C_{\max } \sum_{l=1}^{L} \beta_{l} .}
\end{aligned}
$$

In addition, on event $\mathcal{F}_{3}$, we have

$$
\begin{aligned}
\|\mathbf{Z}[t]\| & =\sqrt{\left\|\mathbf{Q}_{\perp}[t]\right\|^{2}+2 \gamma C_{\max } \sum_{l=1}^{L} \beta_{l} T_{l}[t]} \\
& \leq \sqrt{T_{l^{*}[t]}^{2}[t]+2 \gamma C_{\max } L \beta_{l^{*}[t]} T_{l^{*}[t]}[t]} \\
& \leq \sqrt{T_{l^{*}[t]}^{2}[t]+2 \gamma C_{\max } L T_{l^{*}[t]}[t] \sum_{l=1}^{L} \beta_{l}} \\
& \leq T_{l^{*}[t]}[t] \sqrt{1+2 \gamma C_{\max } L \sum_{l=1}^{L} \beta_{l}}
\end{aligned}
$$

Hence, by utilizing (55) and (56), we have

$$
\begin{aligned}
\|\mathbf{Z}[t+1]\| & \leq\|\mathbf{Z}[t]\|+|\|\mathbf{Z}[t+1]\|-\|\mathbf{Z}[t]\|| \\
& \leq F_{1} T_{l^{*}[t]}[t]+F_{2}
\end{aligned}
$$

where $F_{1} \triangleq L \sqrt{2 \gamma C_{\max } \sum_{l=1}^{L} \beta_{l}}+\sqrt{1+2 \gamma C_{\max } L \sum_{l=1}^{L} \beta_{l}}$ and $F_{2} \triangleq \frac{2 \gamma C_{\max } \sum_{l=1}^{L} \beta_{l}}{\kappa}+2 L \max \left\{A_{\max }, C_{\max }\right\}$. Thus,

$$
\mathbb{E}\left[e^{\eta\|\mathbf{Z}[t+1]\|} ; \mathcal{F}_{3} \mid \mathbf{Q}[t], \mathbf{T}[t]\right] \leq e^{\eta F_{2}} e^{\eta F_{1} T_{l^{*}[t]}[t]}
$$

By substituting (50), (53) and (58) into (47), we have $\mathbb{E}\left[e^{\eta\|\mathbf{Z}[t+1]\|} \mid \mathbf{Q}[t], \mathbf{T}[t]\right] \leq e^{\eta G_{1}}+\rho e^{\eta\|\mathbf{Z}[t]\|}+e^{\eta F_{2}} e^{\eta F_{1} T_{l^{*}[t]}[t]}$ By taking expectation on both sides, we have

$$
\begin{aligned}
& \mathbb{E}\left[e^{\eta\|\mathbf{Z}[t+1]\|}\right] \leq e^{\eta G_{1}}+\rho \mathbb{E}\left[e^{\eta\|\mathbf{Z}[t]\|}\right]+e^{\eta F_{2}} \mathbb{E}\left[e^{\eta F_{1} T_{l^{*}[t]}[t]}\right] \\
& \leq e^{\eta G_{1}}+\rho \mathbb{E}\left[e^{\eta\|\mathbf{Z}[t]\|}\right]+e^{\eta F_{2}} \sum_{l=1}^{L} \mathbb{E}\left[e^{\eta F_{1} T_{l}[t]}\right]
\end{aligned}
$$

Next, we will upper-bound the term $\mathbb{E}\left[e^{\eta F_{1} T_{l}[t]}\right]$.

$$
\begin{aligned}
\mathbb{E}\left[e^{\eta F_{1} T_{l}[t]}\right] & \stackrel{(a)}{=} \sum_{m=0}^{t} e^{\eta F_{1} m} \operatorname{Pr}\left\{T_{l}[t]=m\right\} \\
& \leq \sum_{m=0}^{t} e^{\eta F_{1} m} \operatorname{Pr}\left\{T_{l}[t] \geq m\right\} \\
& \leq \sum_{m=0}^{(b)} e^{\eta F_{1} m} \vartheta^{m} \\
& \leq \sum_{m=0}^{\infty} e^{\eta F_{1} m} \vartheta^{m} \\
& \stackrel{(c)}{=} \frac{1}{1-e^{\eta F_{1}} \vartheta},
\end{aligned}
$$

where the step $(a)$ uses the fact that $T_{l}[t] \leq t$ for any $t \geq 0$; (b) follows from Lemma 2; (c) is true for $0<\eta<\eta_{2}$ and 
$\vartheta e^{\eta_{2} F_{1}}<1$.

By substituting (60) into (59), we have

$$
\mathbb{E}\left[e^{\eta\|\mathbf{Z}[t+1]\|}\right] \leq \rho \mathbb{E}\left[e^{\eta\|\mathbf{Z}[t]\|}\right]+G,
$$

holding for $0<\eta<\eta_{0} \triangleq \min \left\{\eta_{1}, \eta_{2}\right\}$, where $G \triangleq e^{\eta G_{1}}+$ $\frac{L e^{\eta F_{2}}}{1-e^{\eta F_{1} \vartheta}}$. By using (61) and iterating over $t$, we have

$$
\mathbb{E}\left[e^{\eta\|\mathbf{Z}[t]\|]} \leq \rho^{t} e^{\eta\|\mathbf{Z}[0]\|}+\frac{1-\rho^{t}}{1-\rho} G .\right.
$$

Letting $t \rightarrow \infty$ on both sides of the above inequality, we have

$$
\mathbb{E}\left[e^{\eta\left\|\left(\overline{\mathbf{Q}}_{\perp}, \sqrt{2 \gamma C_{\max } \boldsymbol{\beta} \cdot \overline{\mathbf{T}}}\right)\right\|}\right] \leq \frac{G}{1-\rho},
$$

which implies the desired result.

\section{APPENDIX E}

PROOF OF LEMMA 5

$$
\text { If }\|\mathbf{Z}[t]\|>\kappa \text {, then }
$$

$$
\begin{aligned}
& |\|\mathbf{Z}[t+1]\|-\|\mathbf{Z}[t]\|| \\
= & \frac{\left|\|\mathbf{Z}[t+1]\|^{2}-\|\mathbf{Z}[t]\|^{2}\right|}{\|\mathbf{Z}[t+1]\|+\|\mathbf{Z}[t]\|} \\
\leq & \frac{\left|\left\|\mathbf{Q}_{\perp}[t+1]\right\|^{2}-\left\|\mathbf{Q}_{\perp}[t]\right\|^{2}\right|}{\|\mathbf{Z}[t+1]\|+\|\mathbf{Z}[t]\|} \\
& +\frac{2 \gamma C_{\max }\left|\sum_{l=1}^{L} \beta_{l} T_{l}[t+1]-\sum_{l=1}^{L} \beta_{l} T_{l}[t]\right|}{\|\mathbf{Z}[t+1]\|+\|\mathbf{Z}[t]\|} \\
\leq & \frac{\left|\left\|\mathbf{Q}_{\perp}[t+1]\right\|^{2}-\left\|\mathbf{Q}_{\perp}[t]\right\|^{2}\right|}{\left\|\mathbf{Q}_{\perp}[t+1]\right\|+\left\|\mathbf{Q}_{\perp}[t]\right\|} \\
& +\frac{2 \gamma C_{\max }\left|\sum_{l=1}^{L} \beta_{l}-\sum_{l \in \mathbf{H}^{*}} \beta_{l}-\sum_{l \in \mathbf{H}^{*}} \beta_{l} T_{l}[t]\right|}{\|\mathbf{Z}[t+1]\|+\|\mathbf{Z}[t]\|} \\
\leq & \left|\left\|\mathbf{Q}_{\perp}[t+1]\right\|-\left\|\mathbf{Q}_{\perp}[t]\right\|\right| \\
& +\frac{2 \gamma C_{\max }\left(\sum_{l=1}^{L} \beta_{l}-\sum_{l \in \mathbf{H}^{*}} \beta_{l}\right)+2 \gamma C_{\max } \sum_{l \in \mathbf{H}^{*}} \beta_{l} T_{l}[t]}{\|\mathbf{Z}[t+1]\|+\|\mathbf{Z}[t]\|} \\
\leq & \left|\left\|\mathbf{Q}_{\perp}[t+1]\right\|-\left\|\mathbf{Q}_{\perp}[t]\right\|\right|+\frac{2 \gamma C_{\max } \sum_{l=1}^{L} \beta_{l}}{\kappa} \\
& +2 \gamma C_{\max } \frac{\sum_{l \in \mathbf{H}^{*}} \beta_{l} T_{l}[t]}{\sqrt{\left\|\mathbf{Q}_{\perp}[t]\right\|^{2}+2 \gamma C_{\max } \sum_{l=1}^{L} \beta_{l} T_{l}[t]}} .
\end{aligned}
$$

Note that

$$
\begin{aligned}
& \\
& \left|\left\|\mathbf{Q}_{\perp}[t+1]\right\|-\left\|\mathbf{Q}_{\perp}[t]\right\|\right| \\
& \stackrel{(a)}{\leq}\left\|\mathbf{Q}_{\perp}[t+1]-\mathbf{Q}_{\perp}[t]\right\| \\
& \stackrel{(b)}{=}\left\|\mathbf{Q}[t+1]-\mathbf{Q}[t]-\mathbf{Q}_{\|}[t+1]+\mathbf{Q}_{\|}[t]\right\| \\
& \leq\|\mathbf{Q}[t+1]-\mathbf{Q}[t]\|+\left\|\mathbf{Q}_{\|}[t+1]-\mathbf{Q}_{\|}[t]\right\| \\
& \stackrel{(c)}{\leq} 2\|\mathbf{Q}[t+1]-\mathbf{Q}[t]\| \\
& \stackrel{(d)}{\leq} 2\|\mathbf{Q}[t+1]-\mathbf{Q}[t]\|_{1} \\
& \stackrel{\leq}{\leq} L \max _{l}\left|Q_{l}[t+1]-Q_{l}[t]\right| \\
& \leq 2 L \max _{\max }\left\{A_{\max }, C_{\max }\right\},
\end{aligned}
$$

where the step (a) uses the inequality $\left|\|\mathbf{x}\|_{2}-\|\mathbf{y}\|_{2}\right| \leq \| \mathbf{x}-$ $\mathbf{y} \|_{2}$ for any vector $\mathbf{x}$ and $\mathbf{y} ;(b)$ follows from the definition of $\mathbf{Q}=\mathbf{Q}_{\perp}+\mathbf{Q}_{\|} ;(c)$ follows from the non-expansive property of the projection onto the a convex set; $(d)$ is true since $\|\mathbf{x}\| \leq$ $\|\mathbf{x}\|_{1}$ for any vector $\mathbf{x}$. By substituting (63) into (62), we have the desired result.

\section{APPENDIX F}

PROOF OF LEMMA 4

$$
\begin{aligned}
\mathbb{E}\left[X^{n}\right]=\frac{1}{\eta^{n}} \mathbb{E}\left[\left(\log e^{\eta X}\right)^{n}\right] & \stackrel{(a)}{\leq} \frac{1}{\eta^{n}} \mathbb{E}\left[\left(\log \left(e^{n-1} e^{\eta X}\right)\right)^{n}\right] \\
& \stackrel{(b)}{\leq} \frac{1}{\eta^{n}}\left(\log \left(e^{n-1} \mathbb{E}\left[e^{\eta X}\right]\right)\right)^{n},
\end{aligned}
$$

where the step $(a)$ follows from the fact that $f(y)=(\log y)^{n}$ is increasing in $y \in[1, \infty)$ for $n=1,2, \cdots ;(b)$ uses the fact that $g(y)=\left(\log \left(e^{n-1} y\right)\right)^{n}$ is concave in $[1, \infty)$ for $n=$ $1,2, \cdots$, and Jensen's Inequality.

\section{APPENDIX G \\ PRoOF OF INEQUALITY (18)}

To show inequality (18), we need the following lemma.

Lemma 6: Let

$\pi_{\mathbf{c}} \triangleq \operatorname{Pr}\left\{\left\langle\mathbf{d}, \mathbf{c} \cdot \mathbf{S}^{*}(\overline{\mathbf{Q}}, \overline{\mathbf{T}}, \mathbf{c})\right\rangle=b_{\mathbf{c}} \mid \mathbf{C}=\mathbf{c}\right\}$ and

$\chi_{\mathbf{c}} \triangleq \min \left\{b_{\mathbf{c}}-\langle\mathbf{d}, \mathbf{r}\rangle:\right.$ for all $\left.\mathbf{r} \in \mathcal{S}^{(\mathbf{c})} \backslash\left\{\mathbf{w}: b_{\mathbf{c}}=\langle\mathbf{d}, \mathbf{w}\rangle\right\}\right\}$.

Then, for each channel state $\mathbf{c} \in \mathcal{C}$, and any $\epsilon \in\left(0, \chi_{\mathbf{c}} \psi_{\mathbf{c}}\right)$, we have

$$
1-\pi_{\mathbf{c}} \leq \frac{\epsilon}{\chi_{\mathbf{c}} \psi_{\mathbf{c}}},
$$

where we recall that $\psi_{\mathbf{c}} \triangleq \operatorname{Pr}\{\mathbf{C}=\mathbf{c}\}$.

The proof mainly follows from the stability condition, i.e., $\mathbb{E}\left[\left\langle\mathbf{d}, \mathbf{S}^{*}(\overline{\mathbf{Q}}, \overline{\mathbf{T}}, \mathbf{c})\right\rangle\right] \geq\langle\mathbf{d}, \boldsymbol{\lambda}\rangle=b-\epsilon$, and is similar to that of [4, Claim 1]. We omit the proof here for conciseness. Lemma 6 implies that

$$
\begin{aligned}
& \mathbb{E}\left[\left(b_{\mathbf{c}}-\left\langle\mathbf{d}, \mathbf{C} \cdot \mathbf{S}^{*}(\overline{\mathbf{Q}}, \overline{\mathbf{T}}, \mathbf{C})\right\rangle\right)^{2} \mid \mathbf{C}=\mathbf{c}\right] \\
= & \mathbb{E}\left[\left(b_{\mathbf{c}}-\left\langle\mathbf{d}, \mathbf{C} \cdot \mathbf{S}^{*}(\overline{\mathbf{Q}}, \overline{\mathbf{T}}, \mathbf{C})\right\rangle\right)^{2} \mid\left\langle\mathbf{d}, \mathbf{C} \cdot \mathbf{S}^{*}(\overline{\mathbf{Q}}, \overline{\mathbf{T}}, \mathbf{C})\right\rangle \neq b_{\mathbf{c}}\right] \\
& \times\left(1-\pi_{\mathbf{c}}\right) \\
\leq & \frac{\epsilon}{\chi_{\mathbf{c}} \psi_{\mathbf{c}}}\left(\left(b_{\mathbf{c}}\right)^{2}+\left\langle\mathbf{d}, C_{\max } \mathbf{1}\right\rangle^{2}\right) .
\end{aligned}
$$

Similarly, we have

$$
\mathbb{E}\left[b_{\mathbf{c}}-\left\langle\mathbf{d}, \mathbf{C} \cdot \mathbf{S}^{*}(\overline{\mathbf{Q}}, \overline{\mathbf{T}}, \mathbf{C})\right\rangle \mid \mathbf{C}=\mathbf{c}\right] \leq \frac{\epsilon b_{\mathbf{c}}}{\chi_{\mathbf{c}} \psi_{\mathbf{c}}} .
$$


For $\mathbb{E}\left[\left\langle\mathbf{d}, \mathbf{A}-\mathbf{C} \cdot \mathbf{S}^{*}(\overline{\mathbf{Q}}, \overline{\mathbf{T}}, \mathbf{C})\right\rangle^{2} \mid \mathbf{C}=\mathbf{c}\right]$, we have

$$
\begin{aligned}
& \mathbb{E}\left[\left\langle\mathbf{d}, \mathbf{A}-\mathbf{C} \cdot \mathbf{S}^{*}(\overline{\mathbf{Q}}, \overline{\mathbf{T}}, \mathbf{C})\right\rangle^{2} \mid \mathbf{C}=\mathbf{c}\right] \\
= & \mathbb{E}\left[\left(\langle\mathbf{d}, \mathbf{A}\rangle-b+b-b_{\mathbf{c}}+b_{\mathbf{c}}-\left\langle\mathbf{d}, \mathbf{c} \cdot \mathbf{S}^{*}(\overline{\mathbf{Q}}, \overline{\mathbf{T}}, \mathbf{c})\right\rangle\right)^{2}\right] \\
= & \mathbb{E}\left[(\langle\mathbf{d}, \mathbf{A}\rangle-b)^{2}\right]+\left(b-b_{\mathbf{c}}\right)^{2} \\
& +\mathbb{E}\left[\left(b_{\mathbf{c}}-\left\langle\mathbf{d}, \mathbf{c} \cdot \mathbf{S}^{*}(\overline{\mathbf{Q}}, \overline{\mathbf{T}}, \mathbf{c})\right\rangle\right)^{2}\right] \\
& +2(\langle\mathbf{d}, \boldsymbol{\lambda}\rangle-b)\left(b-b_{\mathbf{c}}\right) \\
& +2(\langle\mathbf{d}, \boldsymbol{\lambda}\rangle-b) \mathbb{E}\left[b_{\mathbf{c}}-\left\langle\mathbf{d}, \mathbf{c} \cdot \mathbf{S}^{*}(\overline{\mathbf{Q}}, \overline{\mathbf{T}}, \mathbf{c})\right\rangle\right] \\
& +2\left(b-b_{\mathbf{c}}\right) \mathbb{E}\left[b_{\mathbf{c}}-\left\langle\mathbf{d}, \mathbf{c} \cdot \mathbf{S}^{*}(\overline{\mathbf{Q}}, \overline{\mathbf{T}}, \mathbf{c})\right\rangle\right] .
\end{aligned}
$$

Next, we give upper bounds for each individual term in the right hand side of (67). We will repeatedly use the identity

$$
\langle\mathbf{d}, \boldsymbol{\lambda}\rangle-b=-\epsilon,
$$

where it follows from the definition of $\boldsymbol{\lambda}^{(0)}$ (see Fig. 2). By noting that $b_{\mathbf{c}} \triangleq \max _{\mathbf{s} \in \mathcal{S}^{(\mathbf{c})}}\langle\mathbf{d}, \mathbf{c} \cdot \mathbf{s}\rangle$, we have

$$
\begin{aligned}
& (\langle\mathbf{d}, \boldsymbol{\lambda}\rangle-b) \mathbb{E}\left[b_{\mathbf{c}}-\left\langle\mathbf{d}, \mathbf{c} \cdot \mathbf{S}^{*}(\overline{\mathbf{Q}}, \overline{\mathbf{T}}, \mathbf{c})\right\rangle\right] \\
= & -\epsilon \mathbb{E}\left[b_{\mathbf{c}}-\left\langle\mathbf{d}, \mathbf{c} \cdot \mathbf{S}^{*}(\overline{\mathbf{Q}}, \overline{\mathbf{T}}, \mathbf{c})\right\rangle\right] \leq 0 .
\end{aligned}
$$

In addition, by using inequality (66), we have

$$
\begin{gathered}
\left(b-b_{\mathbf{c}}\right) \mathbb{E}\left[b_{\mathbf{c}}-\left\langle\mathbf{d}, \mathbf{c} \cdot \mathbf{S}^{*}(\overline{\mathbf{Q}}, \overline{\mathbf{T}}, \mathbf{c})\right\rangle\right] \\
\leq b \mathbb{E}\left[b_{\mathbf{c}}-\left\langle\mathbf{d}, \mathbf{c} \cdot \mathbf{S}^{*}(\overline{\mathbf{Q}}, \overline{\mathbf{T}}, \mathbf{c})\right\rangle\right] \leq b \frac{\epsilon b_{\mathbf{c}}}{\chi_{\mathbf{c}} \psi_{\mathbf{c}}} .
\end{gathered}
$$

For $\mathbb{E}\left[(\langle\mathbf{d}, \mathbf{A}\rangle-b)^{2}\right]$, we have

$$
\begin{aligned}
& \mathbb{E}\left[(\langle\mathbf{d}, \mathbf{A}\rangle-b)^{2}\right] \\
= & \mathbb{E}\left[(\langle\mathbf{d}, \mathbf{A}\rangle-\langle\mathbf{d}, \boldsymbol{\lambda}\rangle+\langle\mathbf{d}, \boldsymbol{\lambda}\rangle-b)^{2}\right] \\
= & \mathbb{E}\left[(\langle\mathbf{d}, \mathbf{A}\rangle-\langle\mathbf{d}, \boldsymbol{\lambda}\rangle-\epsilon)^{2}\right] \\
= & \left\langle\mathbf{d}^{2},\left(\boldsymbol{\sigma}^{(\epsilon)}\right)^{2}\right\rangle+\epsilon^{2} .
\end{aligned}
$$

Thus, by substituting (65), (68), (69), (70) and (71) into (67), we have

$$
\begin{aligned}
& \mathbb{E}\left[\left\langle\mathbf{d}, \mathbf{A}-\mathbf{C} \cdot \mathbf{S}^{*}(\overline{\mathbf{Q}}, \overline{\mathbf{T}}, \mathbf{C})\right\rangle^{2} \mid \mathbf{C}=\mathbf{c}\right] \\
\leq & \left\langle\mathbf{d}^{2},\left(\boldsymbol{\sigma}^{(\epsilon)}\right)^{2}\right\rangle+\epsilon^{2}+\left(b-b_{\mathbf{c}}\right)^{2} \\
& +\frac{\epsilon}{\chi_{\mathbf{c}} \psi_{\mathbf{c}}}\left(2 b b_{\mathbf{c}}+\left(b_{\mathbf{c}}\right)^{2}+\left\langle\mathbf{d}, C_{\max } \mathbf{1}\right\rangle^{2}\right)-2 \epsilon\left(b-b_{\mathbf{c}}\right) .
\end{aligned}
$$

By taking the expectation on both sides of the above inequality, we have the desired result.

\section{APPENDIX H}

PRoof OF INEQUALITY (21)

Let $\mathbf{R}^{*}(\mathbf{Q}[t], \mathbf{T}[t]) \triangleq \mathbb{E}\left[\mathbf{C} \cdot \mathbf{S}^{*}(\mathbf{Q}[t], \mathbf{T}[t], \mathbf{C}[t]) \mid \mathbf{Q}[t], \mathbf{T}[t]\right]$. For the face $\mathcal{F} \triangleq \mathcal{H}^{(\mathbf{d})} \cap \mathcal{R}$ of the region $\mathcal{R}$, there exists an angle $\theta \in\left(0, \frac{\pi}{2}\right]$ such that $\left\langle\mathbf{d}, \mathbf{R}^{*}(\mathbf{Q}, \mathbf{T})\right\rangle=b$, for all $\mathbf{Q}$ and
T satisfying $\frac{\|(\mathbf{Q}+\gamma \boldsymbol{\beta} \cdot \mathbf{T})\| \|}{\|\mathbf{Q}+\gamma \boldsymbol{\beta} \cdot \mathbf{T}\|} \geq \cos (\theta)$. Note that

$$
\begin{aligned}
& \mathbb{E}\left[\langle\mathbf{d}, \overline{\mathbf{Q}}\rangle\left\langle\mathbf{d}, \mathbf{C} \cdot \mathbf{S}^{*}(\overline{\mathbf{Q}}, \overline{\mathbf{T}}, \mathbf{C})-\mathbf{A}\right\rangle\right] \\
= & \mathbb{E}\left[\langle\mathbf{d}, \overline{\mathbf{Q}}+\gamma \boldsymbol{\beta} \cdot \overline{\mathbf{T}}\rangle\left\langle\mathbf{d}, \mathbf{C} \cdot \mathbf{S}^{*}(\overline{\mathbf{Q}}, \overline{\mathbf{T}}, \mathbf{C})-\mathbf{A}\right\rangle\right] \\
& -\mathbb{E}\left[\langle\mathbf{d}, \gamma \boldsymbol{\beta} \cdot \overline{\mathbf{T}}\rangle\left\langle\mathbf{d}, \mathbf{C} \cdot \mathbf{S}^{*}(\overline{\mathbf{Q}}, \overline{\mathbf{T}}, \mathbf{C})-\mathbf{A}\right\rangle\right]
\end{aligned}
$$

For $\mathbb{E}\left[\langle\mathbf{d}, \overline{\mathbf{Q}}+\gamma \boldsymbol{\beta} \cdot \overline{\mathbf{T}}\rangle\left\langle\mathbf{d}, \mathbf{C} \cdot \mathbf{S}^{*}(\overline{\mathbf{Q}}, \overline{\mathbf{T}}, \mathbf{C})-\mathbf{A}\right\rangle\right]$, we have

$$
\begin{aligned}
& \mathbb{E}\left[\langle\mathbf{d}, \overline{\mathbf{Q}}+\gamma \boldsymbol{\beta} \cdot \overline{\mathbf{T}}\rangle\left\langle\mathbf{d}, \mathbf{C} \cdot \mathbf{S}^{*}(\overline{\mathbf{Q}}, \overline{\mathbf{T}}, \mathbf{C})-\mathbf{A}\right\rangle\right] \\
= & \mathbb{E}[\langle\mathbf{d}, \overline{\mathbf{Q}}+\gamma \boldsymbol{\beta} \cdot \overline{\mathbf{T}}\rangle(b-\langle\mathbf{d}, \mathbf{A}\rangle)] \\
& -\mathbb{E}\left[\langle\mathbf{d}, \overline{\mathbf{Q}}+\gamma \boldsymbol{\beta} \cdot \overline{\mathbf{T}}\rangle\left(b-\left\langle\mathbf{d}, \mathbf{C} \cdot \mathbf{S}^{*}(\overline{\mathbf{Q}}, \overline{\mathbf{T}}, \mathbf{C})\right\rangle\right)\right]
\end{aligned}
$$

By using the fact that the arrivals are independent of system state, we have

$$
\begin{aligned}
& \mathbb{E}[\langle\mathbf{d}, \overline{\mathbf{Q}}+\gamma \boldsymbol{\beta} \cdot \overline{\mathbf{T}}\rangle(b-\langle\mathbf{d}, \mathbf{A}\rangle)] \\
= & \epsilon \mathbb{E}[\langle\mathbf{d}, \overline{\mathbf{Q}}+\gamma \boldsymbol{\beta} \cdot \overline{\mathbf{T}}\rangle] \\
= & \epsilon \mathbb{E}\left[\left\|\overline{\mathbf{Q}}_{\|}\right\|\right]+\gamma \epsilon \mathbb{E}\left[\left\|(\boldsymbol{\beta} \cdot \overline{\mathbf{T}})_{\|}\right\|\right] .
\end{aligned}
$$

For $\mathbb{E}\left[\langle\mathbf{d}, \overline{\mathbf{Q}}+\gamma \boldsymbol{\beta} \cdot \overline{\mathbf{T}}\rangle\left(b-\left\langle\mathbf{d}, \mathbf{C} \cdot \mathbf{S}^{*}(\overline{\mathbf{Q}}, \overline{\mathbf{T}}, \mathbf{C})\right\rangle\right)\right]$, we have

$$
\begin{aligned}
& \mathbb{E}\left[\langle\mathbf{d}, \overline{\mathbf{Q}}+\gamma \boldsymbol{\beta} \cdot \overline{\mathbf{T}}\rangle\left(b-\left\langle\mathbf{d}, \mathbf{C} \cdot \mathbf{S}^{*}(\overline{\mathbf{Q}}, \overline{\mathbf{T}}, \mathbf{C})\right\rangle\right)\right] \\
= & \mathbb{E}\left[\mathbb{E}\left[\left\|(\overline{\mathbf{Q}}+\gamma \boldsymbol{\beta} \cdot \overline{\mathbf{T}})_{\|}\right\|\left(b-\left\langle\mathbf{d}, \mathbf{C} \cdot \mathbf{S}^{*}(\overline{\mathbf{Q}}, \overline{\mathbf{T}}, \mathbf{C})\right\rangle\right) \mid \overline{\mathbf{Q}}, \overline{\mathbf{T}}\right]\right] \\
= & \mathbb{E}\left[\left\|(\overline{\mathbf{Q}}+\gamma \boldsymbol{\beta} \cdot \overline{\mathbf{T}})_{\|}\right\|\left(b-\left\langle\mathbf{d}, \mathbf{R}^{*}(\overline{\mathbf{Q}}, \overline{\mathbf{T}})\right\rangle\right)\right] \\
\stackrel{(a)}{=} & \mathbb{E}\left[\|\overline{\mathbf{Q}}+\gamma \boldsymbol{\beta} \cdot \overline{\mathbf{T}}\| \cos (\phi)\left(b-\left\langle\mathbf{d}, \mathbf{R}^{*}(\overline{\mathbf{Q}}, \overline{\mathbf{T}})\right\rangle\right)\right] \\
\stackrel{(b)}{=} & \mathbb{E}\left[\|\overline{\mathbf{Q}}+\gamma \boldsymbol{\beta} \cdot \overline{\mathbf{T}}\| \cos (\phi) \mathbb{1}_{\{\phi>\theta\}}\left(b-\left\langle\mathbf{d}, \mathbf{R}^{*}(\overline{\mathbf{Q}}, \overline{\mathbf{T}})\right\rangle\right)\right] \\
\stackrel{(c)}{=} & \mathbb{E}\left[\left\|(\overline{\mathbf{Q}}+\gamma \boldsymbol{\beta} \cdot \overline{\mathbf{T}})_{\perp}\right\| \cot (\phi) \mathbb{1}_{\{\phi>\theta\}}\left(b-\left\langle\mathbf{d}, \mathbf{R}^{*}(\overline{\mathbf{Q}}, \overline{\mathbf{T}})\right\rangle\right)\right] \\
\stackrel{(d)}{\leq} & \cot (\theta) \mathbb{E}\left[\left\|(\overline{\mathbf{Q}}+\gamma \boldsymbol{\beta} \cdot \overline{\mathbf{T}})_{\perp}\right\|\left(b-\left\langle\mathbf{d}, \mathbf{R}^{*}(\overline{\mathbf{Q}}, \overline{\mathbf{T}})\right\rangle\right)\right] \\
\stackrel{(e)}{\leq} & \cot (\theta) \sqrt{\mathbb{E}\left[\left\|(\overline{\mathbf{Q}}+\gamma \boldsymbol{\beta} \cdot \overline{\mathbf{T}})_{\perp}\right\|^{2}\right] \mathbb{E}\left[\left(b-\left\langle\mathbf{d}, \mathbf{R}^{*}(\overline{\mathbf{Q}}, \overline{\mathbf{T}})\right\rangle\right)^{2}\right]} \\
\leq & \cot (\theta) \sqrt{\mathbb{E}\left[\|\left(\overline{\mathbf{Q}} \perp\left\|^{2}+\gamma^{2}\right\| \boldsymbol{\beta} \cdot \overline{\mathbf{T}} \|^{2}\right]\right.} \\
& \times \sqrt{\mathbb{E}\left[\left(b-\left\langle\mathbf{d}, \mathbf{R}^{*}(\overline{\mathbf{Q}}, \overline{\mathbf{T}})\right\rangle\right)^{2}\right]},
\end{aligned}
$$

where the step $(a)$ is true for that $\phi$ is the angle between vector $\overline{\mathbf{Q}}+\gamma \boldsymbol{\beta} \cdot \overline{\mathbf{T}}$ and the normal vector $\mathbf{d} ;(b)$ follows from the definition of $\theta ;(c)$ uses $\left\|(\overline{\mathbf{Q}}+\gamma \boldsymbol{\beta} \cdot \overline{\mathbf{T}})_{\perp}\right\|=\| \overline{\mathbf{Q}}+\gamma \boldsymbol{\beta}$. $\overline{\mathbf{T}} \| \sin (\phi) ;(d)$ follows from the fact that cotangent function is decreasing in $\left(0, \frac{\pi}{2}\right] ;(e)$ uses Cauchy-Schwartz Inequality.

Next, let's consider $\mathbb{E}\left[\left(b-\left\langle\mathbf{d}, \mathbf{R}^{*}(\overline{\mathbf{Q}}, \overline{\mathbf{T}})\right\rangle\right)^{2}\right]$.

$$
\begin{aligned}
& \mathbb{E}\left[\left(b-\left\langle\mathbf{d}, \mathbf{R}^{*}(\overline{\mathbf{Q}}, \overline{\mathbf{T}})\right\rangle\right)^{2}\right] \\
& \stackrel{(a)}{=} \mathbb{E}\left[\left(\mathbb{E}\left[\Psi-\left\langle\mathbf{d}, \mathbf{C} \cdot \mathbf{S}^{*}(\overline{\mathbf{Q}}, \overline{\mathbf{T}}, \mathbf{C})\right\rangle \mid \overline{\mathbf{Q}}, \overline{\mathbf{T}}\right]\right)^{2}\right] \\
& \stackrel{(b)}{\leq} \mathbb{E}\left[\mathbb{E}\left[\left(\Psi-\left\langle\mathbf{d}, \mathbf{C} \cdot \mathbf{S}^{*}(\overline{\mathbf{Q}}, \overline{\mathbf{T}}, \mathbf{C})\right\rangle\right)^{2} \mid \overline{\mathbf{Q}}, \overline{\mathbf{T}}\right]\right] \\
&= \mathbb{E}\left[\left(\Psi-\left\langle\mathbf{d}, \mathbf{C} \cdot \mathbf{S}^{*}(\overline{\mathbf{Q}}, \overline{\mathbf{T}}, \mathbf{C})\right\rangle\right)^{2}\right] \\
&= \sum_{\mathbf{c} \in \mathcal{C}} \psi_{\mathbf{c}} \mathbb{E}\left[\left(b_{\mathbf{c}}-\left\langle\mathbf{d}, \mathbf{C} \cdot \mathbf{S}^{*}(\overline{\mathbf{Q}}, \overline{\mathbf{T}}, \mathbf{C})\right\rangle\right)^{2} \mid \mathbf{C}=\mathbf{c}\right] \\
& \stackrel{(c)}{\leq} \epsilon \sum_{\mathbf{c} \in \mathcal{C}} \frac{1}{\chi_{\mathbf{c}}}\left(\left(b_{\mathbf{c}}\right)^{2}+\left\langle\mathbf{d}, C_{\max } \mathbf{1}\right\rangle^{2}\right),
\end{aligned}
$$


where the step $(a)$ follows from the definition of $\Psi$ with distribution $\operatorname{Pr}\left\{\Psi=b_{\mathbf{c}}\right\}=\psi_{\mathbf{c}}$ for $\mathbf{c} \in \mathcal{C}$, and the definition of $\mathbf{R}^{*}(\overline{\mathbf{Q}}, \overline{\mathbf{T}})$; (b) uses Jensen's Inequality; (c) uses (65).

Thus, by substituting (74), (75) and (76) into (73), we have

$$
\begin{aligned}
& \mathbb{E}\left[\langle\mathbf{d}, \overline{\mathbf{Q}}+\gamma \boldsymbol{\beta} \cdot \overline{\mathbf{T}}\rangle\left\langle\mathbf{d}, \mathbf{C} \cdot \mathbf{S}^{*}(\overline{\mathbf{Q}}, \overline{\mathbf{T}}, \mathbf{C})-\mathbf{A}\right\rangle\right] \\
\geq & \epsilon \mathbb{E}\left[\left\|\overline{\mathbf{Q}}_{\|}\right\|\right]+\gamma \epsilon \mathbb{E}\left[\left\|(\boldsymbol{\beta} \cdot \overline{\mathbf{T}})_{\|}\right\|\right] \\
& -\cot (\theta) \sqrt{\left(\mathbb{E}\left[\left\|\overline{\mathbf{Q}}_{\perp}\right\|^{2}\right]+\gamma^{2} \mathbb{E}\left[\|\boldsymbol{\beta} \cdot \overline{\mathbf{T}}\|^{2}\right]\right) \epsilon} \\
& \times \sqrt{\sum_{\mathbf{c} \in \mathcal{C}} \frac{1}{\chi_{\mathbf{c}}}\left(\left(b_{\mathbf{c}}\right)^{2}+\left\langle\mathbf{d}, C_{\max } \mathbf{1}\right\rangle^{2}\right)} .
\end{aligned}
$$

For $\mathbb{E}\left[\langle\mathbf{d}, \gamma \boldsymbol{\beta} \cdot \overline{\mathbf{T}}\rangle\left\langle\mathbf{d}, \mathbf{C} \cdot \mathbf{S}^{*}(\overline{\mathbf{Q}}, \overline{\mathbf{T}}, \mathbf{C})-\mathbf{A}\right\rangle\right]$, we have

$$
\begin{aligned}
& \stackrel{(a)}{\leq} \mathbb{E}[\langle\mathbf{d}, \gamma \boldsymbol{\beta} \cdot \overline{\mathbf{T}}\rangle(b-\langle\mathbf{d}, \boldsymbol{\lambda}\rangle)] \\
& \stackrel{(b)}{=} \gamma \in \mathbb{E}\left[\left\|(\boldsymbol{\beta} \cdot \overline{\mathbf{T}})_{\|}\right\|\right],
\end{aligned}
$$$$
\mathbb{E}\left[\langle\mathbf{d}, \gamma \boldsymbol{\beta} \cdot \overline{\mathbf{T}}\rangle\left\langle\mathbf{d}, \mathbf{C} \cdot \mathbf{S}^{*}(\overline{\mathbf{Q}}, \overline{\mathbf{T}}, \mathbf{C})-\mathbf{A}\right\rangle\right]
$$$$
=\mathbb{E}\left[\mathbb{E}\left[\langle\mathbf{d}, \gamma \boldsymbol{\beta} \cdot \overline{\mathbf{T}}\rangle\left\langle\mathbf{d}, \mathbf{C} \cdot \mathbf{S}^{*}(\overline{\mathbf{Q}}, \overline{\mathbf{T}}, \mathbf{C})-\mathbf{A}\right\rangle \mid \overline{\mathbf{Q}}, \overline{\mathbf{T}}\right]\right]
$$$$
=\mathbb{E}\left[\mathbb{E}\left[\langle\mathbf{d}, \gamma \boldsymbol{\beta} \cdot \overline{\mathbf{T}}\rangle\left(\left\langle\mathbf{d}, \mathbf{R}^{*}(\overline{\mathbf{Q}}, \overline{\mathbf{T}})\right\rangle-\langle\mathbf{d}, \mathbf{A}\rangle\right) \mid \overline{\mathbf{Q}}, \overline{\mathbf{T}}\right]\right]
$$$$
=\mathbb{E}\left[\langle\mathbf{d}, \gamma \boldsymbol{\beta} \cdot \overline{\mathbf{T}}\rangle\left(\left\langle\mathbf{d}, \mathbf{R}^{*}(\overline{\mathbf{Q}}, \overline{\mathbf{T}})\right\rangle-b+b-\langle\mathbf{d}, \boldsymbol{\lambda}\rangle\right)\right]
$$

where the step $(a)$ uses the fact that $\left\langle\mathbf{d}, \mathbf{R}^{*}(\overline{\mathbf{Q}}, \overline{\mathbf{T}})\right\rangle \leq b ;(b)$ uses (68). By substituting (77) and (78) into (72), we have the desired result.

\section{REFERENCES}

[1] M. Bramson. State space collapse with application to heavy traffic limits for multiclass queueing networks. Queueing Systems, 30(1):89-140, 1998.

[2] L. Bui, R. Srikant, and A. Stolyar. Novel architectures and algorithms for delay reduction in back-pressure scheduling and routing. In Proc. IEEE International Conference on Computer Communications (INFOCOM), Rio de Janeiro, Brazil, April, 2009.

[3] A. Eryilmaz and R. Srikant. Fair resource allocation in wireless networks using queue-length based scheduling and congestion control. In Proc. IEEE International Conference on Computer Communications (INFOCOM), Miami, Florida, March 2005.

[4] A. Eryilmaz and R. Srikant. Asymptotically tight steady-state queue length bounds implied by drift conditions. Queueing Systems, 72:311$359,2012$.

[5] G. Foschini and J. Salz. A basic dynamic routing problem and diffusion. IEEE Transactions on Communications, 26(3):320-327, 1978.

[6] G. R. Gupta and N. Shroff. Delay analysis for multi-hop wireless networks. In Proc. IEEE International Conference on Computer Communications (INFOCOM), Rio de Janeiro, Brazil, April 2009.

[7] B. Hajek. Hitting-time and occupation-time bounds implied by drift analysis with applications. Advances in Applied Probability, 14(3):502$525,1982$.

[8] I. Hou, V. Borkar, and P. R. Kumar. A theory of QoS for wireless. In Proc. IEEE International Conference on Computer Communications (INFOCOM), Rio de Janeiro, Brazil, April, 2009.

[9] J. Jaramillo, R. Srikant, and L. Ying. Scheduling for optimal rate allocation in ad hoc networks with heterogeneous delay constraints. IEEE Journal on Selected Areas in Communications, 29(5):979-987, 2011.

[10] B. Ji, C. Joo, and N. B. Shroff. Delay-based back-pressure scheduling in multihop wireless networks. Networking, IEEE/ACM Transactions on, 21(5):1539-1552, 2013.

[11] B. Li and A. Eryilmaz. Optimal distributed scheduling under timevarying conditions: A Fast-CSMA algorithm with applications. IEEE Transactions on Wireless Communications, 12(7):3278-3288, 2013.

[12] B. Li, R. Li, and A. Eryilmaz. Heavy-traffic-optimal scheduling design with regular service guarantees in wireless networks. In Proc. ACM international symposium on Mobile ad hoc networking and computing (MOBIHOC), July 2013.
[13] B. Li, R. Li, and A. Eryilmaz. Throughput-optimal scheduling design with regular service guarantees in wireless networks. Accepted by IEEE/ACM on Networking, 2014. DOI: 10.1109/TNET.2014.2333008.

[14] R. Li, A. Eryilmaz, and B. Li. Throughput-optimal scheduling with regulated inter-service times. In Proc. IEEE International Conference on Computer Communications (INFOCOM), Turin, Italy, April 2013.

[15] X. Lin and N. Shroff. Joint rate control and scheduling in multihop wireless networks. In Proceedings of IEEE Conference on Decision and Control, Paradise Island, Bahamas, December 2004.

[16] N. McKeown, A. Mekkittikul, V. Anantharam, and J. Walrand. Achieving $100 \%$ throughput in an input-queued switch. Communications, IEEE Transactions on, 47(8):1260-1267, 1999.

[17] M. Neely, E. Modiano, and C. Li. Fairness and optimal stochastic control for heterogeneous networks. In Proc. IEEE International Conference on Computer Communications (INFOCOM), Miami, Florida, March 2005.

[18] D. Shah and D. Wischik. Switched networks with maximum weight policies: Fluid approximation and multiplicative state space collapse. Annals of Applied Probability, 22(1):70-127, 2012.

[19] A. Stolyar. Maxweight scheduling in a generalized switch: State space collapse and workload minimization in heavy traffic. The Annals of Applied Probability, 14(1):1-53, 2004.

[20] A. Stolyar. Maximizing queueing network utility subject to stability: Greedy primal-dual algorithm. Queueing Systems, 50(4):401-457, 2005.

[21] L. Tassiulas and A. Ephremides. Stability properties of constrained queueing systems and scheduling policies for maximum throughput in multihop radio networks. IEEE Transactions on Automatic Control, 36(12):1936-1948, 1992.

[22] W. Whitt. Weak convergence theorems for priority queues: Preemptiveresume discipline. Journal of Applied Probability, 8(1):74-94, 1971.

[23] R. Williams. Diffusion approximations for open multiclass queueing networks: sufficient conditions involving state space collapse. Queueing Systems, 30(1):27-88, 1998.

[24] H. Xiong, R. Li, A. Eryilmaz, and E. Ekici. Delay-aware cross-layer design for network utility maximization in multi-hop networks. Selected Areas in Communications, IEEE Journal on, 29(5):951 -959, May 2011.

[25] L. Ying, S. Shakkottai, and A. Reddy. On combining shortest-path and back-pressure routing over multihop wireless networks. In Proc. IEEE International Conference on Computer Communications (INFOCOM), Rio de Janeiro, Brazil, April 2009.

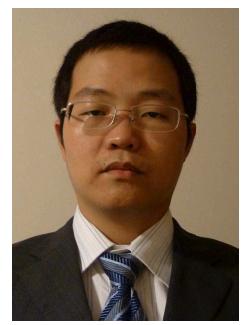

Bin Li (S'12-M'15) received his B.S. degree in Electronic and Information Engineering in 2005, M.S. degree in Communication and Information Engineering in 2008, both from Xiamen University, China, and Ph.D. degree in Electrical and Computer Engineering from The Ohio State University in May 2014. He is currently a Postdoctoral Researcher in the Coordinated Science Laboratory at the University of Illinois at Urbana-Champaign.

His research interests include wireless communication and networks, cloud computing, resource allocation and management, distributed algorithm design, queueing theory, and optimization theory. He received the Presidential Fellowship from The Ohio State University and Chinese Government Award for Outstanding Ph.D. Students Abroad in 2013

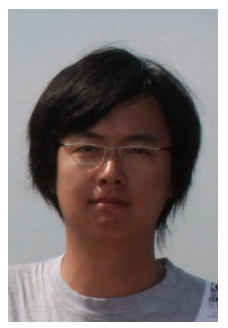

Ruogu Li (S'10) received his B.S. degree in Electronic Engineering from Tsinghua University, Beijing, in 2007. He received his PhD degree in Electrical and Computer Engineering from Ohio State University. His research interests include optimal network control, wireless communication networks, low-delay scheduling scheme design and cross-layer algorithm design. His $\mathrm{PhD}$ dissertation discussed the principles and methods of adaptive network algorithm design under various quality-of-service requirements. He is now a staff system engineer at ASSIA Inc. in Redwood City, California. 


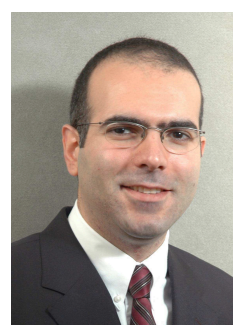

Atilla Eryilmaz (S'00-M '06) received his B.S. degree in Electrical and Electronics Engineering from Bogaziçii University, Istanbul, in 1999, and the M.S. and Ph.D. degrees in Electrical and Computer Engineering from the University of Illinois at Urbana-Champaign in 2001 and 2005, respectively. Between 2005 and 2007, he worked as a Postdoctoral Associate at the Laboratory for Information and Decision Systems at the Massachusetts Institute of Technology. He is currently an Associate Professor of Electrical and Computer Engineering at The Ohio State University, where he has been a faculty since 2007 . He served as a TPC chair for the International Symposium on Modeling and Optimization in Mobile, Ad Hoc and Wireless Networks (WiOpt) 2015, and is an Associate Editor for ACM/IEEE Transactions on Networking since March 2015.

Dr. Eryilmaz's research interests include design and analysis for complex networked systems with focus on wireless communication and power networks, optimal control of stochastic networks, optimization theory, distributed algorithms, network pricing, and information theory. He was a co-author of the Best Student Paper Award in WiOpt 2012. He received the NSF-CAREER Award in 2010, and two Lumley Research Awards for Research Achievements in 2010 and 2015. 